\title{
Semi-classical Stark broadening calculations of Hel lines in a non-ideal plasma
}

\author{
H. Ben Chaouacha ${ }^{1}$, S. Sahal-Bréchot ${ }^{2}$, and N. Ben Nessib ${ }^{3}$
}

\author{
1 Institut Préparatoire aux Études d'Ingénieur El Manar, Campus Universitaire, BP No 244 Manar II, 2092 Tunis, Tunisia \\ ${ }^{2}$ Laboratoire d'Étude du Rayonnement et de la Matière en Astrophysique, UMR CNRS 8112-LERMA, Observatoire de Paris, \\ Section de Meudon, 92195 Meudon Cedex, France \\ e-mail: Sylvie.Sahal-Brechot@obspm.fr \\ 3 Groupe de Recherche en Physique Atomique et Astrophysique, Institut National des Sciences Appliquées et de Technologie, \\ Centre Urbain Nord, BP No 676, 1080 Tunis Cedex, Tunisia
}

Received 12 July 2006 / Accepted 11 December 2006

\section{ABSTRACT}

\begin{abstract}
New semi-classical collision functions are used to compute the inelastic contribution to the impact electronic total width, by considering three interaction potentials (Coulomb Debye, Cut-off, and Ion Sphere). Numerical results are calculated for the neutral helium $6678 \AA\left(2^{1} \mathrm{P}^{\circ}-3^{1} \mathrm{D}\right)$ and $5876 \AA\left(2^{3} \mathrm{P}^{\circ}-3^{3} \mathrm{D}\right)$ visible lines. The lines corresponding to these transitions are isolated, and the plasma is weakly non-ideal for all temperatures and electronic densities of interest. For electronic collisions, semi-classical perturbation theory is sufficient and the impact approximation is well satisfied. The ion effects can be treated within the quasistatic approximation, and the quasistatic ionic contribution is dominated by the polarization (or quadratic) $r_{\mathrm{p}}^{-4}$-interaction. To consider both the electron and the ion effects, the microfield distributions and the complete reduced Stark profile of isolated line are calculated using different methods. The computed total widths corresponding to the three interaction potentials are compared to the available experimental widths and then approximated by appropriate formulae. The data we obtained provide an opportunity to test various approximations included in the semi-classical perturbation formalism. They are also of interest for stellar spectroscopic diagnosis in dense atmospheres (white dwarfs for instance).
\end{abstract}

Key words. atomic data - line: profiles - atomic processes

\section{Introduction}

Stark broadening of spectral lines by interactions of radiating atoms or ions with perturbing electrons is of great importance for a number of astrophysics applications. It is found to be a reliable tool for characterizing strongly coupled plasmas that have become experimentally more accessible in recent years. This requires, in practice, a detailed knowledge of the various governing atomic processes, especially for dense plasmas where the screening effects are not negligible (Song \& Jung 2003). To describe the interactions between the perturbing electrons and the emitting atoms in these non-ideal plasmas, we may use either the Cut-off $V^{\mathrm{C}}$ (Ben Nessib et al. 1997) or the Ion Sphere models $V^{\mathrm{IS}}$ (Salzmann \& Szichman. 1987; Gutierrez 1994; Jung \& Yoon 2000a), where appropriate corrective terms for the Coulomb potential are introduced.

In a previous paper (Ben Chaouacha et al. 2004), we modified the standard formalism of Stark impact broadening of spectral lines by using the cut-off $V^{\mathrm{C}}$ and the ion sphere $V^{\mathrm{IS}}$ interaction potential instead of the Coulomb-Debye $V^{\mathrm{CD}}$ one, which should be more appropriate at high densities. Thus we have derived new semi-classical collision functions for both the transition probability and the cross section in the case of electron-atom collisions. These new functions take into account the plasma screening effects by introducing a reliable cut-off in the interaction potential when the electron-atom distance exceeds a certain radius. They will be used here in order to compute the inelastic contribution to the impact electronic total width: the upper cut-off at $R_{\mathrm{D}}$ (Debye length) for the Coulomb Debye model has been replaced by $R_{\mathrm{c}}$ (mean distance between particles) for the Cut-off and the Ion Sphere models; see below.

The purpose of this paper is to validate our theoretical approach. Our numerical results will be compared to experimental widths. Helium is selected as an example because of its importance in stellar spectra, and because it is a simple atomic system so that the wave functions are calculated with fair accuracy (Griem 1964, 1974; Konjević et al. 2002).

In a recent paper, Omar et al. (2006) investigated Stark widths of isolated neutral helium lines at electron densities less than $5 \times 10^{17} \mathrm{~cm}^{-3}$ using thermodynamic Green functions.

A detailed analysis of the broadening of helium spectral lines of dense plasmas can be used to test our understanding of correlation effects in these plasmas. The cases of the $6678 \AA$ $\left(2{ }^{1} \mathrm{P}^{\circ}-3^{1} \mathrm{D}\right)$ and $5876 \AA\left(2^{3} \mathrm{P}^{\circ}-3^{3} \mathrm{D}\right) \mathrm{HeI}$ visible lines are chosen by considering the same conditions of densities $N_{\mathrm{e}}$ and temperatures $T$ as the experiments of Gauthier et al. (1981) and Bücher et al. (1995), and an upper cut-off at $R_{\mathrm{D}}$ for the Coulomb Debye model and at $R_{\mathrm{c}}$ for the Cut-off and the Ion Sphere models.

The impact inelastic electronic widths $W_{\mathrm{e}, \text { inel }}^{\mathrm{CD}}, W_{\mathrm{e}, \text { inel }}^{\mathrm{C}}$ and $W_{\mathrm{e}, \text { inel }}^{\mathrm{IS}}$ are obtained with the three different potentials, but the contribution of the elastic electronic collisions $W_{\mathrm{e}, \mathrm{el}}^{\mathrm{CD}}$ will be calculated only with the Coulomb Debye model. Since the very long-range collisions are not important for the elastic contribution, it is expected to be relatively weak for this line. In addition, it will be possible to consider that the results for the elastic 
contribution should not be very different in the case of the Cutoff and the Ion Sphere potentials.

For the studied lines, the contribution of the collisions with the ions of the plasma is important and cannot be ignored. It is quasistatic at the considered densities, that are rather high $(4 \times$ $10^{17} \mathrm{~cm}^{-3}<N_{\mathrm{e}}<9 \times 10^{17} \mathrm{~cm}^{-3}$, for the $6678 \AA$ line). The adiabatic assumption is always valid for the ion broadening of isolated lines. The quasistatic ionic contribution is dominated by the polarization (or quadratic) $r_{\mathrm{p}}^{-4}$-interaction.

To consider both the ionic and the electronic effects, the total width will be obtained from the Full Width at Half Maximum (FWHM) deduced from the complete reduced Stark profile $j_{A, \mathrm{r}}(x)$ of isolated lines. The profile depends on the Debye shielding parameter $r$ and the quasistatic quadratic ion broadening parameter $A$ which must be related to the impact electronic total width; the associated microfield distribution $W_{\mathrm{r}}$ will be calculated with: (a) Baranger \& Mozer's method (1959, 1960); (b) Hooper's method (Hooper 1966, 1968a,b); and (c) the Analytic Fitting Formulas (AFF) method (Potekhin et al. 2002).

The computed total widths $W_{\text {tot }}^{\mathrm{CD}}, W_{\text {tot }}^{\mathrm{C}}$ and $W_{\text {tot }}^{\mathrm{IS}}$ will be compared to the experimental widths $W^{\exp }$ (Gauthier et al. 1981; Bücher et al. 1995).

The results obtained in the present paper provide an opportunity to test various approximations included in the semi-classical perturbation formalism.

This paper is divided into five sections. In Sect. 2, we give some theoretical background with the basic assumptions and equations regarding: i) the non-ideality factor $\gamma$ and the plasma classification; ii) the isolated line approximation; iii) the validity criteria of the impact approximation; iv) the Stark broadening impact theory of isolated lines; v) the main interaction potentials describing the non-ideal plasmas; vi) the collision functions associated with the inelastic cross-section and the transition probability; and vii) the numerical methods used to derive the electrical microfield distributions. The validity criteria of our investigated theoretical approach, as well as the applicability range of the main governing parameters, are discussed in this section. Section 3 deals with the description of the numerical method used to calculate the different contributions to the width. Section 4 discusses the effects of the parameters that govern the different contributions to the width calculated for the considered lines.

Then, we compare the three total widths $W_{\text {tot }}^{\mathrm{CD}}, W_{\text {tot }}^{\mathrm{C}}$ and $W_{\text {tot }}^{\mathrm{IS}}$ with the experimental widths $W^{\exp }$ relative to the same transitions (Gauthier et al. 1981; Bücher et al. 1995), and approximate them with appropriate formulae. Section 5 summarizes the main results and gives some related open problems.

\section{Theoretical background}

We present here a brief summary of the theory because Stark broadening impact theory has been extensively developed since the fundamental work by Baranger (1958a,b,c).

\subsection{Non-ideality factor $\gamma$}

Plasmas may be classified into different types, depending on their temperature $T$ and their electronic density $N_{\mathrm{e}}$ (Günther et al. 1985; Ben Nessib et al. 1997). A plasma is ideal if the interactions between particles of the medium can be neglected. Non-ideality may be due to charge-charge, charge-neutral and neutral-neutral interactions. At extremely high densities, atomic valence electrons are shared by other atoms; such a dense plasma is similar to liquid metals, though its free electrons are not necessarily degenerate. This type of plasma is called non-ideal, strongly coupled, non-Debye, or simply dense plasma.

Non-ideal plasmas cover the range of densities between gases and solids. These may be obtained by gas plasma compression or by extension and heating of solids and liquids. In these conditions, the mean interaction potential energy $E_{\mathrm{p}}$ between charged particles is not small compared with their kinetic energy $E_{\mathrm{k}}$.

Contrary to low pressure plasmas, where the kinetic energy $E_{\mathrm{k}}$ of a particle is always high in comparison to the mean interaction potential energy $E_{\mathrm{p}}$ between two neighboring charged particles, in dense plasmas it is of the same order of magnitude or even lower than $E_{\mathrm{p}}$. Thus, the so-called non-ideality parameter $\gamma$ that characterizes this behavior:

$\gamma=\frac{E_{\mathrm{p}}}{E_{\mathrm{k}}}$

is expected to be greater than unity $(\gamma \geq 1)$.

At high neutral density, non-ideality due to neutrals is also possible, but if we restrict ourselves only to charged-particle interaction, the potential energy $E_{\mathrm{p}}$ may be expressed as (Vitel et al. 1990):

$E_{\mathrm{p}}=\frac{Z^{2} e^{2}}{4 \pi \varepsilon_{0} r_{0}}$

where the radius $r_{0}$ is related to the ion charge $Z$ and the electron concentration $N_{\mathrm{e}}$ by:

$r_{0}=\left(\frac{3 Z}{4 \pi N_{\mathrm{e}}}\right)^{\frac{1}{3}}$

The velocity average $\bar{v}$ involved in the electron kinetic energy $\left(E_{\mathrm{k}}=\frac{1}{2} m_{\mathrm{e}} \bar{v}^{2}\right)$, is expressed as (Freudenstein 1978):

$\bar{v}=\left(3 k_{\mathrm{B}} T / m_{\mathrm{e}}\right)^{1 / 2}$

which is expected to be convenient, because the most important contribution to the widths comes from high velocities due to the properties of the collision functions $a(z)$. The non-ideality factor $\gamma$ is expressed as follows:

$\gamma=2.69310^{-3} \frac{\left(N_{\mathrm{e}}\right)^{1 / 3}}{T}$

The electronic density $N_{\mathrm{e}}$ is expressed in $\mathrm{cm}^{-3}$ and the plasma temperature $T$ in Kelvin. For a constant plasma density $N_{\mathrm{e}}$, the plasma temperature $T$ decreases when the non-ideality factor $\gamma$ increases. Hence, the main physical characteristics are expected to be significantly different from one plasma type to another, which needs systematically different theoretical and experimental approaches to study their spectral responses.

Depending on the value of $\gamma$, the considered plasma may be qualified either as ideal, weakly coupled, coupled, or strongly coupled. Unfortunately, a $\gamma$-range relative to each of these plasma types is not yet precisely defined in the literature, which may be ascribed mainly to the lack of sufficient experimental data, especially for both the coupled and strongly coupled plasmas. In modern plasma experiments, $\gamma$ may approach unity, whereas in stellar matter it can be much larger. In these cases, correlations between plasmas particles should not be neglected.

Table 1 gives the values of the non-ideal factor $\gamma$ for the studied lines, calculated by considering the conditions of densities $N_{\mathrm{e}}$ and temperatures $T$ of the experiments of Gauthier et al. (1981) 
Table 1. Validity criteria for the isolated line approximation and the impact approximation, calculated for the considered HeI transitions. $\gamma$ : nonideality factor $-N_{\mathrm{e}} / N_{\mathrm{l}}$ : ratio of the electronic density to the maximum electron density for which the line may be considered as isolated $-C_{\text {impact }}^{\mathrm{e}}$ : impact approximation validity criterion for the electronic collisions $-C_{\text {impact }}^{\mathrm{i}}$ : impact approximation validity criterion for the ionic collisions $C_{\text {impact }}^{\mathrm{s}, \mathrm{e}}:$ impact approximation validity criterion for the strong electronic collisions $-C_{\text {impact }}^{\mathrm{s}, \mathrm{i}}$ : impact approximation validity criterion for the strong ionic collisions.

\begin{tabular}{ccccccccc}
\hline \hline Transition & $\begin{array}{c}T \\
\left(10^{4} \mathrm{~K}\right)\end{array}$ & $\begin{array}{c}N_{\mathrm{e}} \\
\left(10^{17} \mathrm{~cm}^{-3}\right)\end{array}$ & $\gamma$ & $N_{\mathrm{e}} / N_{\mathrm{l}}$ & $\begin{array}{c}C_{\text {impact }}^{\mathrm{e}} \\
\left(10^{-3}\right)\end{array}$ & $\begin{array}{c}C_{\text {impact }}^{\mathrm{i}} \\
(2.0\end{array}$ & $\begin{array}{c}C_{\text {impact }}^{\mathrm{s,e},} \\
\left(10^{-3}\right)\end{array}$ & $C_{\text {impact }}^{\mathrm{s}, \mathrm{i}}$ \\
\hline$\left(2 \mathrm{P}^{\circ}-3^{1} \mathrm{D}\right)$ & 1.35 & 2.0 & 0.116 & 0.19 & 38.3 & 3.72 & 4.7 & 2.0 \\
$6678 \AA$ & 1.78 & 3.3 & 0.104 & 0.29 & 45.0 & 5.42 & 5.5 & 3.0 \\
& 1.86 & 4.9 & 0.114 & 0.35 & 59.5 & 7.65 & 7.9 & 4.4 \\
& 1.93 & 6.2 & 0.119 & 0.44 & 68.6 & 9.14 & 9.4 & 5.5 \\
& 2.01 & 6.9 & 0.118 & 0.46 & 72.3 & 9.92 & 10.1 & 6.0 \\
& 2.13 & 8.4 & 0.119 & 0.56 & 79.8 & 11.34 & 11.4 & 7.1 \\
& 2.09 & 8.9 & 0.124 & 0.58 & 84.8 & 11.76 & 12.4 & 7.7 \\
& 2.28 & 10.0 & 0.118 & 0.64 & 86.4 & 12.82 & 12.6 & 8.3 \\
& 2.32 & 10.7 & 0.119 & 0.68 & 89.8 & 13.41 & 13.2 & 8.8 \\
& 2.40 & 11.8 & 0.119 & 0.72 & 94.0 & 14.27 & 14.0 & 9.5 \\
& 2.44 & 13.2 & 0.121 & 0.79 & 100.6 & 15.13 & 15.4 & 10.5 \\
& 2.59 & 13.7 & 0.116 & 0.81 & 99.0 & 15.79 & 14.9 & 10.7 \\
$\left(2{ }^{3} \mathrm{P}^{\circ}-3^{3} \mathrm{D}\right)$ & 2.55 & 14.9 & 0.121 & 0.91 & 106.4 & 16.27 & 16.5 & 11.6 \\
$5876 \AA$ & 4.46 & 4.9 & 0.157 & 0.07 & 19.4 & 0.80 & 3.0 & 0.4 \\
& 5.20 & 10.2 & 0.200 & 0.15 & 34.7 & 1.55 & 5.5 & 0.8 \\
& 5.21 & 12.0 & 0.211 & 0.18 & 40.3 & 1.82 & 6.4 & 0.9 \\
& 5.60 & 18.4 & 0.244 & 0.26 & 56.5 & 2.70 & 9.2 & 1.4 \\
& 6.35 & 24.9 & 0.270 & 0.35 & 67.9 & 3.44 & 11.1 & 1.8 \\
\hline
\end{tabular}

and Bücher et al. (1995). As $\gamma \approx 0.12$ for the $6678 \AA$ line and $\gamma<0.3$ for the $5876 \AA$ line, the considered plasmas are weakly non-ideal for these densities.

Another physical parameter allowing a quantitative comparison of the different plasma types is the number of particles in the Debye sphere, $N_{\mathrm{D}}$, which is defined by:

$N_{\mathrm{D}}=\frac{4}{3} \pi R_{\mathrm{D}}^{3}\left(N_{\mathrm{e}}+N_{\mathrm{i}}\right)$

where $N_{\mathrm{i}}=N_{\mathrm{e}}$ designates the ion density and $R_{\mathrm{D}}$ the Debye radius, which is expressed by:

$R_{\mathrm{D}}=\left(\frac{k_{\mathrm{B}} T}{4 \pi N_{\mathrm{e}} e^{2}}\right)^{\frac{1}{2}}$.

Weakly non-ideal means that there is approximately one charged particle in the Debye sphere $\left(N_{\mathrm{D}}=1\right)$. Strongly non-ideal means that there are about one tenth or fewer particles in the Debye sphere. This condition implies that the Debye theory is no longer valid.

\subsection{Isolated line approximation}

It is not necessary to discuss uncertainties arising from all the approximations involved in the broadening calculations, since the criteria for their application are given in detail elsewhere (SahalBréchot 1969a,b). However, we recall the conditions of validity for the isolated line approximation (Sahal-Bréchot 1969a,b; Dimitrijević \& Sahal-Bréchot 1984a,b).

A line is isolated if non-degenerate energy levels broadened by collisions do not overlap. Denoting by $2 w_{i}$ and $2 w_{f}$ the corresponding level widths, we can express the specified conditions by:

$2 w_{i} \leq \omega_{i i^{\prime}}, \quad 2 w_{f} \leq \omega_{f f^{\prime}}$, where $\omega_{j j^{\prime}}(j=i, f)$ is the energy distance to the nearest perturbing level $\left[\omega_{j j^{\prime}}=\min \left(\omega_{i i^{\prime}}, \omega_{f f^{\prime}}\right)\right]$. If $w \leq \omega_{j j^{\prime}}$, where $w$ is the half-width of the line, the line can be considered as isolated. Thus, if we want to make certain that the line is isolated, we must verify that (Dimitrijević \& Sahal-Bréchot 1984a,b):

$2 w(\AA) \leq C / 10^{16}$,

where

$C=10^{8} \lambda^{2}(\AA)\left[\left(E_{j}-E_{j^{\prime}}\right)\left(\mathrm{cm}^{-1}\right)\right]$.

If half-widths are available for a certain electron density $N_{\mathrm{e}}$ (e.g., $10^{16} \mathrm{~cm}^{-3}$, as reported in Dimitrijević \& Sahal-Bréchot 1984a,b, 1990), an electron density $N_{1}\left(\mathrm{~cm}^{-3}\right)$ is defined as:

$N_{\mathrm{l}}=\frac{C}{2 w(\AA)} \times \frac{N_{\mathrm{e}}}{10^{16}}$.

So, for an electron density lower than $N_{\mathrm{l}}$, the line can be treated as isolated in the core, even if weak forbidden components due to the failure of this approximation still appear in the wings (Dimitrijević \& Sahal-Bréchot 1984b). If $\frac{N_{\mathrm{e}}}{N_{\mathrm{l}}}>1$ we have to treat the problem of overlapping lines.

For the $6678 \AA$ line, the energy distance between the upper level $\left(3^{1} \mathrm{D}\right)$ and its nearest perturbing level $\left(3^{1} \mathrm{P}^{\circ}\right)$ is $\Delta E_{j j^{\prime}}=$ $100 \mathrm{~cm}^{-1}$, so $C=4.5 \times 10^{17}$, while for the $5876 \AA$ line, $\Delta E_{j j^{\prime}}=$ $537 \mathrm{~cm}^{-1}$ and $C=1.8 \times 10^{18}$. Table 1 shows that the ratio $\frac{N_{\mathrm{e}}}{N_{\mathrm{l}}}$ is less than unity, especially when the temperature decreases. Thus the lines corresponding to these transitions are well isolated in these conditions.

\subsection{Validity criteria of the Impact approximation}

As already discussed by Baranger (1958c, 1962) and Sahal-Bréchot (1969a,b), the impact (or binary) approximation 
is valid when the average effect of collisions is weak, or equivalently when strong collisions are separated in time. Following Baranger (1958a,b,c), the condition of validity of the impact approximation has been rederived by Ben Nessib et al. (1996):

$C_{\text {impact }}=N \times \pi \frac{4}{3} \rho_{\text {typ }}^{3} \sim N \pi \rho_{\text {typ }}^{3} \ll 1$,

where $\rho_{\text {typ }}=\tau_{\text {typ }} \bar{v}$ is a typical impact parameter for strong collisions and $N$ is the density of the perturbers.

A typical value of the impact parameter $\rho_{\text {typ }}$ can be obtained by

$2 w=N \bar{v} \pi \rho_{\text {typ }}^{2}$.

The mean duration of a typical collision, the so-called collision time $\tau_{\text {typ }}$, must be very much smaller than the mean interval $\Delta T=\frac{1}{2 w}$ between two collisions:

$\tau_{\text {typ }}=\frac{\rho_{\text {typ }}}{\bar{v}} \ll \frac{1}{N \bar{v} \pi \rho_{\text {typ }}^{2}}$,

that gives:

$N \pi \rho_{\text {typ }}^{3} \ll 1$.

This means that the collision volume $\pi \frac{4}{3} \rho_{\text {typ }}^{3}$ must be very small compared to the inverse of the density of the perturbers $N$. In other words, there is only one particle at the same time in the collision volume. This condition is well verified by electronic collisions for a large range of densities. However, at high densities $\left(N_{\mathrm{e}} \geq 10^{18} \mathrm{~cm}^{-3}\right)$, one must check if the validity criterion of the electron-impact approximation $(\rho w / \bar{v}) \ll 1$ is fulfilled or not. This is a general problem of dense plasmas, since the criterion cannot remain fulfilled with increasing densities for a constant temperature (Griem et al. 1962; Griem 1974).

If the plasma is strongly non-ideal (high density and low temperature), $\rho_{\text {typ }}$ is of the order of the thermal de Broglie length $\lambda=\frac{\hbar}{m_{\mathrm{e}} \bar{v}}$. Thus, for strongly non-ideal plasmas, the validity criterion for the impact approximation can be written as follows (Ben Nessib et al. 1997)

$T N_{\mathrm{e}}^{-2 / 3}>\left(\frac{4 \pi}{3}\right)^{2 / 3} \frac{\pi \hbar^{2}}{8 m_{\mathrm{e}} k_{\mathrm{B}}}$.

So, if the temperature is expressed in Kelvin and the density in $\mathrm{cm}^{-3}$, this condition becomes $T N_{\mathrm{e}}^{-2 / 3}>9.02 \times 10^{-12}$.

This allows Ben Nessib et al. (1997) to construct a diagram representing the different plasma conditions, which shows that there is a region where the impact approximation is valid for a strongly non-ideal plasma.

Table 1 shows the impact validity criteria for the two studied lines, by considering the same conditions of densities $N_{\mathrm{e}}$ and temperatures $T$ as the experiments of Gauthier et al. (1981) and Bücher et al. (1995) and the Coulomb Debye model with an upper cut-off at $R_{\mathrm{D}}$.

For the $5876 \AA$ line, the impact approximation is always fulfilled for collisions with electrons: the corresponding value of $C_{\text {impact }}^{\mathrm{e}}$ is small compared to unity. For the $6678 \AA$ line, $C_{\text {impact }}^{\mathrm{e}}$ is of the order of 0.1 which corresponds to the limit of the impact approximation. As reported in Gauthier et al. (1981), the impact approximation for the treatment of the electrons breaks down at densities greater than $10^{18} \mathrm{~cm}^{-3}$.
Since the strong electronic collisions are well separated in time (for the $6678 \AA$ line, $C_{\text {impact }}^{\mathrm{s}, \mathrm{e}}$ are of the order of $5 \times 10^{-3}$ to $10^{-2}$ ), the impact approximation remains valid. Weak collisions can be treated by the perturbation theory, and thus their contributions are additive, allowing us to treat them with the impact theory (Baranger 1958a,b,c, 1962), even if $C_{\text {impact }}^{\mathrm{e}}$ is not very small compared to unity.

Concerning collisions of neutral atoms with ions, the impact approximation is most often valid in the physical conditions of hot stellar atmospheres where the density is weak $\left(10^{10}\right.$ to $10^{14} \mathrm{~cm}^{-3}$ ) and where the ion perturbers are light, such as protons and $\mathrm{He}^{+}$ions. For example, at $N_{\mathrm{e}}=10^{13} \mathrm{~cm}^{-3}$, one obtains $C_{\text {impact }}^{\mathrm{i}}=1,66 \times 10^{-4}$. In laboratory experiments, the density is higher $\left(10^{16}-10^{18} \mathrm{~cm}^{-3}\right)$ and the perturbers are heavier $\left(\mathrm{Ar}^{+}\right.$ for instance). At medium densities $\left(10^{16} \mathrm{~cm}^{-3}\right)$, ion dynamics effects are generally not negligible and have to be considered (Ben Nessib et al. 1996). At high densities $\left(10^{17} \mathrm{~cm}^{-3}\right.$ and more), the impact approximation breaks down and the quasistatic approximation must be applied. In the studied experiment, the density is high $\left(10^{18} \mathrm{~cm}^{-3}\right.$ and more $)$ and the quasistatic approximation is reliable. We will show in Sect. 3 that the quadratic interaction is predominant and the quadrupolar one is negligible.

\subsection{Stark broadening impact theory of isolated lines}

The semi-classical description has been extensively used for Stark broadening calculations and for collisional transition probabilities entering the statistical equilibrium equations for nonLocal Thermodynamical Equilibrium (N-LTE) studies. The calculation procedure is well described elsewhere (Sahal-Bréchot $1969 a, b)$. We recall the basic formulae leading to the evaluation of electron impact-broadening parameters of isolated spectral lines, within the framework of semi-classical perturbational formalism.

Within the impact approximation, the profile is Lorentzian for isolated lines. Overlapping lines are outside the scope of the present study. The following set of equations was used for the computation of the impact total electron width $W_{\mathrm{e}}$ (in angular frequency units).

For the line corresponding to the transition between the initial level $i$ and the final level $f$, the half-width $w$ and the shift $d$ are given by Baranger's formula (Baranger 1958c)

$$
\begin{aligned}
w+\mathrm{i} d= & N_{\mathrm{e}} \int_{0}^{\infty} v f(v) \mathrm{d} v \int_{0}^{\infty} 2 \pi \rho \mathrm{d} \rho \\
& \times\left\{1-\langle\mathrm{i}|S| \mathrm{i}\rangle\left\langle f\left|S^{-1}\right| f\right\rangle\right\}_{A V},
\end{aligned}
$$

where $w+\mathrm{i} d$ is a complex number $\left(\mathrm{i}^{2}=-1\right) . N_{\mathrm{e}}$ denotes the electron density, $S$, the scattering matrix obtained for the atomperturber interaction corresponding to the impact parameter $\rho$ of the incoming electron and the relative velocity $v, f(v)$, the relative atom-perturber Maxwell distribution of velocities, and, $\{\ldots\}_{A V}$, the angular average over the magnetic quantum numbers which will not be detailed here.

Equation (17) is the general result to be used in all cases where electron broadening is treated by the impact approximation. In the derivation by Sahal-Bréchot (1969a,b), the linewidth can be expressed in terms of elastic and inelastic contributions. For the transition between the levels $i\left(n_{i} l_{i} L_{i} S_{i} J_{i}\right)$ and $f\left(n_{f} l_{f} L_{f} S_{f} J_{f}\right)$, the full width at half intensity $W_{\mathrm{e}}=2 w_{\mathrm{e}}$ 
can be put in the form (Sahal-Bréchot 1969a,b; Dimitrijević \& Sahal-Bréchot 1984a,b, 1985)

$W_{\mathrm{e}}=2 w_{\mathrm{e}}=N_{\mathrm{e}} \int_{0}^{\infty} v f(v) \mathrm{d} v \sigma_{t}(v)$,

where the total cross-section $\sigma_{t}(v)$ is defined by

$\sigma_{t}(v)=\sum_{j \neq i} \sigma_{i j}(v)+\sum_{j^{\prime} \neq f} \sigma_{f j^{\prime}}(v)+\sigma_{\mathrm{el}}$,

where $j$ and $j^{\prime}$ respectively denote the levels that perturb $i$ and $f$.

The inelastic cross-sections $\sigma_{i j}(v)$ (resp. $\left.\sigma_{f j^{\prime}}(v)\right)$ are given by an integration over the impact parameter $\rho$ of the transition probabilities $P_{i j}(v, \rho)\left(\right.$ resp. $\left.P_{f j^{\prime}}(v, \rho)\right)$ as

$$
\begin{aligned}
\sum_{j \neq i} \sigma_{i j}(v)= & \pi R_{1}^{2} \sum_{j \neq i} P_{i j}\left(v, R_{1}\right) \\
& +\int_{R_{1}}^{R_{\mathrm{D}}} 2 \pi \rho \mathrm{d} \rho \sum_{j \neq i} P_{i j}(v, \rho) .
\end{aligned}
$$

The expression for $P_{i j}$ (resp. $P_{f j^{\prime}}$ ) is given within the first order time dependent perturbation theory by an average over the initial Zeeman states $M_{i}$ and a sum over the final states $M_{j}$ (Seaton 1962)

$$
\begin{aligned}
P_{i j}(\rho, v) & =\frac{1}{2 J_{i}+1} \sum_{M_{i}, M_{j}} \frac{1}{\hbar^{2}} \\
\times & \left|\int_{-\infty}^{+\infty}\left\langle n_{i} l_{i} J_{i} M_{i}|V(t)| n_{j} l_{j} J_{j} M_{j}\right\rangle \exp \left(\frac{\mathrm{i}\left(E_{j}-E_{i}\right) t}{\hbar}\right) \mathrm{d} t\right|^{2},
\end{aligned}
$$

where $V(t)$ denotes the interaction potential between the atom and the charged perturber moving along a classical path at time $t$, and $E_{i}$ (resp. $E_{j}$ ), the energy of the $i$ (resp. $j$ ) level.

The elastic cross-section $\sigma_{e l}$ is given by

$\sigma_{\mathrm{el}}=2 \pi R_{2}^{2}+\int_{R_{2}}^{R_{\mathrm{D}}} 8 \pi \rho \mathrm{d} \rho \sin ^{2} \delta$,

with

$\delta=\left(\Phi_{\mathrm{p}}^{2}+\Phi_{\mathrm{q}}^{2}\right)^{1 / 2}$,

where the phase-shifts $\Phi_{\mathrm{p}}$ and $\Phi_{\mathrm{q}}$ are respectively due to the polarization potential $\left(\sim r^{-4}\right)$ and the quadrupolar potential $\left(\sim r^{-3}\right)$ parts (Sahal-Bréchot 1969a,b). We refer to the original papers by Sahal-Bréchot (1969a,b) for the choice of the cut-offs $R_{1}$ and $R_{2}$.

The transition probabilities, the cross-sections and the impact parameter are symmetrized, in order to ensure the unitarity and the symmetry of the collision S-matrix. We refer to Seaton (1962), Feautrier (1968) and Sahal-Bréchot (1969a,b) for details of the symmetrization procedures.

\subsection{Interaction potentials in plasmas}

In the standard formalism of Stark impact broadening of spectral lines and of cross sections, the electrostatic Coulomb potential is used to describe the interaction between the perturbing electrons and the emitting atom. It is well known, however, that the extreme conditions of some plasma environments can drastically alter transition rates from their values for the corresponding isolated systems. Long-range Coulomb interactions are screened by plasmas, leading to shorter-range interactions (Stewart \& Pyatt 1966). The effect of the plasma was modelled in these collision studies by static screened interactions, the justification of which requires some rather specific conditions (Weisheit 1984; Scheibner et al. 1987). First, the reciprocal of the electron-ion collision duration, $1 / \tau_{\mathrm{ei}}=v_{\mathrm{ei}}$, must be less than the plasma (electron) frequency, $v_{\mathrm{e}}=\left(\frac{4 \pi e^{2} N_{\mathrm{e}}}{m_{\mathrm{e}}}\right)$. If this condition is not fulfilled, plasma screening of the target may not be accurately represented by a static potential arising from the average electronic charge density in the ion's vicinity. Second, substantial screening of the electronic interaction is achieved only when the threshold energy of the excitation, $\Delta E$, is less than $h v_{\mathrm{e}}$; otherwise the bound electron's motion is too fast to permit screening by most of the free electrons. Furthermore, in the absence of plasma screening, it is known that as the ratio of $k_{\mathrm{B}} T / \Delta E$ increases, the relative importance of ion-impact excitation increases with respect to electron-impact excitation.

In the case of a weakly coupled plasma, the amount of the Coulomb forces in the interaction energy may be defined by the Debye-Hückel theory which corresponds to a classical treatment of the charged particle interactions (Debye \& Hückel 1923). In standard Stark broadening calculations, the following approximation is most often used:

$\left\{\begin{array}{l}V^{\mathrm{CD}}(t)=\frac{Z_{\mathrm{p}} e^{2}(Z+N)}{r_{\mathrm{p}}} \\ -Z_{\mathrm{p}} e^{2} \sum_{i=1}^{N} \frac{1}{r_{i \mathrm{p}}} ; r_{\mathrm{p}} \leq R_{\mathrm{D}} \\ V^{\mathrm{CD}}(t)=0 ; r_{\mathrm{p}}>R_{\mathrm{D}}\end{array}\right.$

where $r_{\mathrm{p}}$ denotes the distance between the perturber electron and the emitter center, $r_{\mathrm{ip}}$ is the distance between the perturber and a bound atomic electron, $Z_{\mathrm{p}} e$ is the charge of the perturber $\left(Z_{\mathrm{p}}=-1\right.$ for an electron), $Z e$ is charge of the radiating atom ( $Z=0$ for a neutral atom), and $N$ is the number of its electrons. The two-particle Coulomb field is shielded by the ensemble of surrounding electrons: the electronic correlations (screening effects) are taken into account by introducing a cutoff in the interaction when the electron-atom distance exceeds the Debye radius $R_{\mathrm{D}}$.

This Coulomb-Debye potential $V^{\mathrm{CD}}$ is often a good approximation for high temperature and low density plasmas, but it is no longer valid at the limit of low temperatures and high densities, where the mean electrostatic interaction energy is much greater in magnitude than the mean kinetic energy of the ions. For these non-ideal plasma conditions, the Coulomb Cut-off potential $V^{\mathrm{C}}$ is more suited to describe the interaction between the perturber and the emitter, since it adds a corrective term as follows (Scheibner et al. 1987):

$$
\left\{\begin{array}{l}
V^{\mathrm{C}}(t)=\frac{Z_{\mathrm{p}} e^{2}(Z+N)}{r_{\mathrm{p}}} \\
-Z_{\mathrm{p}} e^{2} \sum_{i=1}^{N} \frac{1}{r_{\mathrm{ip}}}\left(1-\frac{r_{\mathrm{p}}}{R_{\mathrm{c}}}\right) ; r_{\mathrm{p}} \leq R_{\mathrm{c}} \\
V^{\mathrm{C}}(t)=0 ; r_{\mathrm{p}}>R_{\mathrm{c}}
\end{array}\right.
$$

where $R_{\mathrm{c}}$ designates a cut-off parameter assumed to be equal to the Ion Sphere radius $R_{\mathrm{c}}=\left(3 Z / 4 \pi N_{\mathrm{e}}\right)^{1 / 3}$ (Scheibner et al. 1987).

A reasonable model describing the strongly correlated plasmas that is widely used in the literature is one in which each ion of charge $Z$ is surrounded by a sphere of radius $R_{\mathrm{c}}$ containing $Z$ uniformly distributed free electrons. In this Ion Sphere picture, 
two corrective terms are added to the Coulomb model (Salzmann \& Szichman. 1987):

$$
\left\{\begin{array}{l}
V^{\mathrm{IS}}(t)=\frac{Z_{\mathrm{p}} e^{2}(Z+N)}{r_{\mathrm{p}}} \\
-Z_{\mathrm{p}} e^{2} \sum_{i=1}^{N} \frac{1}{r_{\mathrm{ip}}}\left(1-\frac{3}{2} \frac{r_{\mathrm{p}}}{R_{\mathrm{c}}}+\frac{1}{2} \frac{r_{\mathrm{p}}^{3}}{R_{\mathrm{c}}^{3}}\right) ; r_{\mathrm{p}} \leq R_{\mathrm{c}} \\
V^{\mathrm{IS}}(t)=0 ; r_{\mathrm{p}}>R_{\mathrm{c}} .
\end{array}\right.
$$

Several comments about the Ion Sphere potential $V^{\mathrm{IS}}$ can be made. First, for the calculated cross sections to be significant, the binary collision picture must be valid, that is, strong collisions must be separated in time. Second, the Ion Sphere model may represent only the strong screening limit. Moreover, at short distances, electronic overlapping must be considered. Account of these quantum effects has new consequences which may be explained quasi-classically as short-range inter-particle repulsion forces. An effective repulsion is due to electron degeneracy, although that last effect appears at very high densities $(\gamma>1)$. However, under these conditions it becomes very difficult to derive exact expressions for the associated potentials.

\subsection{Collision functions}

In the standard formalism of Stark impact broadening of spectral lines and of cross sections, the Coulomb-Debye potential Eq. (24) is used. In addition, the integration over the time Eq. (22) is performed from $-\infty$ to $+\infty$ and not from $-t_{\mathrm{D}}$ to $+t_{\mathrm{D}}$, $t_{\mathrm{D}}$ being the time when the atom-perturber distance is equal to the Debye length $R_{\mathrm{D}}$. Consequently, an upper cut-off at $R_{\mathrm{D}}$ is introduced at the impact parameter integration stage.

Under this condition, the integration over the time gives the collision function for the transition probability, $A^{\mathrm{CD}}(z)$ (Griem et al. 1962; Sahal-Bréchot 1969a,b):

$A^{\mathrm{CD}}(z)=z^{2}\left[K_{0}^{2}(z)+K_{1}^{2}(z)\right]$.

$K_{0}(z), K_{1}(z)$ designate the modified Bessel functions of the zero and first order, and $z=\frac{\rho \omega_{i j}}{v}$, where $v$ designates the electron perturber velocity, $\rho$, the impact parameter, and $\omega_{i j}=\frac{E_{i}-E_{j}}{\hbar}$, where $E_{i}, E_{j}$ correspond to the energies of the states $i$ and $j$.

The integration of $A^{\mathrm{CD}}(z) / z$ over the impact parameter $z$ gives the collision function for the total inelastic cross-section, $a^{\mathrm{CD}}(z)($ Griem et al. 1962; Sahal-Bréchot 1969a):

$a^{\mathrm{CD}}(z)=z\left[K_{0}(z) K_{1}(z)\right]$.

The corresponding functions for the Cut-off model (Ben Nessib et al. 1997) are denoted by $A^{\mathrm{C}}(z)$ and $a^{\mathrm{C}}(z)$. Their expressions are given in Ben Chaouacha et al. (2004).

The corresponding functions for the Ion Sphere model (Ben Chaouacha et al. 2004, 2005) are denoted by $A^{\mathrm{IS}}(z)$ and $a^{\mathrm{IS}}(z)$. Their expressions are given in Ben Chaouacha et al. (2004, 2005).

As in the standard formalism of the Coulomb-Debye potential, the integration over the time Eq. (23) is performed from $-\infty$ to $+\infty$ and not from $-t_{\mathrm{c}}$ to $+t_{\mathrm{c}}, t_{\mathrm{c}}$ being the time when the atomperturber distance is equal to the $R_{\mathrm{c}}$. Then an upper cut-off at $R_{\mathrm{c}}$ will be introduced at the impact parameter integration stage.

For collisions with electrons, the parameter $z_{\mathrm{c}}=\frac{R_{\mathrm{c}} \omega_{i j}}{v}$ is expressed as (Sahal-Bréchot 1969a,b):

$z_{\mathrm{c}}=\frac{1}{2} \sqrt{\frac{m_{\mathrm{p}}}{m_{\mathrm{e}}}} \frac{\Delta E_{i j}}{I_{\mathrm{H}}} \sqrt{\frac{I_{\mathrm{H}}}{E}} R_{\mathrm{c}}$, where $m_{\mathrm{p}}$ is the proton mass, $m_{\mathrm{e}}$ is the electron mass, $I_{\mathrm{H}}$ is the ionization energy of hydrogen, $\Delta E_{i j}=100 \mathrm{~cm}^{-1}$ for the $6678 \AA$ HeI line $\left(3{ }^{1} \mathrm{P}^{\circ}\right.$ is the nearest perturber level of $\left.3^{1} D\right)$. For $T=$ $40000 \mathrm{~K}$ and $N_{\mathrm{e}}=10^{16} \mathrm{~cm}^{-3}, E=\frac{3}{2} k_{\mathrm{B}} T=42250 \mathrm{~cm}^{-1}$ and $R_{\mathrm{c}}=549 a_{0}$. Thus a typical value for $z_{\mathrm{c}}$ is 0.40 .

\subsection{Electrical microfield distributions}

Because of the Stark effect, stochastic electric microfields have an effect on optical and thermodynamic properties of a plasma. Line shape calculations in turn require as input the electric microfield distribution at the emitting atom or ion.

Various approximate theories have been proposed to evaluate the electric microfield distribution $W_{r}$. In this section, we summarize the basic relations derived in the literature that are used for the calculations presented in this paper.

For a wide class of spectral lines, the observed frequencies (measured from line center) are sufficiently large that the ions in the plasma are effectively stationary over the corresponding radiation time. The emitting atoms or ions in plasmas are under the influence of electric fields produced by relatively rapidly moving electrons and slowly moving ions. This effect is associated with the idea that the electric microfield acting on the test particles is the sum of all the electric fields created by the perturbing ions, on the scale of distances at which the quasineutrality condition is not fulfilled. The radiator is immersed in a statistically fluctuating field produced by the configuration of the plasma during the time of emission; this is assumed short compared to times in which the configuration changes significantly. The Hamiltonian $H(F)$ describing the usual static Stark effect depends on the electric field strength $F$ produced by the perturbing ions. Hence the evaluation of the quasistatic broadening is reduced to determining both the Stark levels of $H(F)$ and the proper statistical distribution function $W(F)$ of the perturbing electric fields.

\subsubsection{Baranger \& Mozer's distribution}

The first calculations were made neglecting all interactions between charged particles (Holtsmark 1919; Margenau \& Lewis 1959). Only the Coulomb electric fields of point ions were considered and assumed to be random and static. Then the distribution function is dependent only on the ion density and may be written as (Griem et al. 1962; Griem 1974):

$W(F)=\frac{1}{F_{0}} W_{\mathrm{H}}\left(\frac{F}{F_{0}}\right)=\frac{1}{F_{0}} W_{\mathrm{H}}(\beta)$,

where the normal field strength $F_{0}$ is expressed by

$F_{0}=\frac{2.61 e}{4 \pi \epsilon_{0}} N_{\mathrm{e}}^{2 / 3} \approx \frac{\left(4 \pi N_{\mathrm{e}} / 3\right)^{2 / 3} e}{4 \pi \epsilon_{0}}$,

for singly charged ions (we assume here that $N_{\mathrm{e}}=N_{\mathrm{i}}$, where $N_{\mathrm{i}}$ is the ion density). When cgs units are used, $4 \pi \epsilon_{0}=1$.

The Holtsmark distribution function $W_{\mathrm{H}}(\beta)$ for the normalized ion field strength $\beta=\frac{F}{F_{0}}$ is (Holtsmark 1919)

$W_{\mathrm{H}}(\beta)=\frac{2}{\pi} \beta \int_{0}^{\infty} \eta \exp \left(-\eta^{3 / 2}\right) \sin (\beta \eta) \mathrm{d} \eta$.

In the calculation of $W_{\mathrm{H}}(\beta)$, it has been implicitly assumed that the perturbing ions were statistically independent and that the electron screening is negligible. This assumption is justified for 
very hot or rarefied plasmas for which the Coulomb coupling parameter $\gamma$ is close to zero. However, at high charged particles densities where Stark broadening is important, interactions between perturbers are not negligible, and the probability of a given configuration of ions depends on the electrostatic interaction energy between the ions. With relation to this problem, various theories of the electric microfield distribution have been formulated that are based mainly on a cluster expansion in powers of density (Baranger \& Mozer 1959; Mozer \& Baranger 1960). The primary aim of these efforts has been to include particleparticle correlations to various orders and thus to improve the original work done by Holtsmark (1919).

The corrected distribution function in which ion-ion correlations and Debye shielding by electrons are taken into account is expressed as (Griem 1974)

$W(F)=\frac{1}{F_{0}} W_{r}\left(\frac{F}{F_{0}}\right)=\frac{1}{F_{0}} W_{r}(\beta)$.

Then the ion field strength distribution function $W_{r}(\beta)$ at neutral points and at singly charged ions is expressed by Griem (1974)

$W_{r}(\beta)=\frac{2}{\pi} \beta \int_{0}^{\infty} \eta A_{r}(\eta) \sin (\beta \eta) \mathrm{d} \eta$.

$W_{r}(\beta)$ depends on the ratio $r$ of the mean distance between the ions $R_{\mathrm{c}}$ and the Debye length $R_{\mathrm{D}}$, i.e.:

$r=R_{\mathrm{c}} / R_{\mathrm{D}}=6^{1 / 3} \pi^{1 / 6}\left(k_{\mathrm{B}} T\right)^{-1 / 2} e N_{\mathrm{e}}^{1 / 6}$,

The $A_{r}(\eta)$ function designates the Fourier transform of $W(F)$ (Griem 1974; Mozer \& Baranger 1960):

$A_{r}(\eta)=\frac{4}{\pi} \int \frac{\exp (\mathrm{i} \eta F) W(F)}{F^{2}} \mathrm{~d} F$.

This function was calculated using an Ursell cluster integral expansion (Mozer \& Baranger 1960) and takes the following form

$A_{r}(\eta)=\exp \left[-\eta^{3 / 2} \chi^{\prime}\left(r \eta^{1 / 2}\right)-\psi^{\prime}\left(r \eta^{1 / 2}\right)\right]$,

which is reduced in the Holtsmark limit with $r=0$ to

$A_{0}(\eta)=\exp \left(-\eta^{3 / 2}\right)$.

The two functions $\chi^{\prime}$ and $\psi^{\prime}$ depend only on the variable $r \eta^{1 / 2}$. They represent, respectively, the contributions of one-body and two-body clusters to the series expansion (Mozer \& Baranger 1960).

\subsubsection{Hooper's distribution}

In order to extend the theory to higher densities and lower temperatures, Hooper (1966, 1968a,b) developed a new method based on a collective-coordinate technique that allows for the inclusion of all correlations to a high degree of accuracy. The plasma is assumed as a system of $N$ singly charged particles moving in a uniform neutralizing background. Each of these $N$ particles interacts with each other through an effective potential which includes the effect of the ion-electron interactions. The plasma is assumed to be in thermal equilibrium and macroscopically neutral. The effect of the uniform neutralizing background may be included by writing the potential energy of the system in terms of its Fourier expansion, which makes a distinction between the Coulomb-Debye model and the correlated models (Hooper 1966, 1968a,b). Then, the expression for the microfield distribution $W_{r}(\beta)$ is written as

$W_{r}(\beta)=\frac{1}{2 \pi} \beta \int_{0}^{\infty} T(t) \sin (\beta t) t \mathrm{~d} t$

To proceed further, this integral is treated by an Ursell cluster expansion technique similar to that employed by Mayer \& Mayer (1940) and Baranger \& Mozer (Baranger \& Mozer 1959; Mozer $\&$ Baranger 1960). Although the general technique is similar, the characteristic functions $T(t)$, in terms of which the expansion is made, are very different from those used by Mayer \& Mayer (1940) and Baranger \& Mozer (Baranger \& Mozer 1959; Mozer $\&$ Baranger 1960). The final result is given in the following.

For the correlated plasmas, the asymptotic $W_{r}(\beta)$ curve can, with a high degree of accuracy, be calculated using only the first approximation to the theory. $T(t)$ may be put into a more convenient form (Hooper 1966, 1968a,b):

$T(t)=\exp \left[-\widetilde{\gamma} L^{2}+I_{1}(t)\right]$

where the functions $\widetilde{\gamma}, L$ and $I_{1}$ depend on the potential model, the shielding parameter $r$ and an arbitrary, real, positive parameter $\widetilde{\alpha}$ which will be independently determined (Hooper 1966 , 1968a,b).

Due to the difficulties of optimizing the adjustable parameter $\widetilde{\alpha}$ (Hooper 1966, 1968a,b), a direct evaluation of the microfield distributions $W_{r}(\beta)$ using this method involves significant effort, particularly when a large number of integrals at different values of $\beta$ are needed. As a result, extensive numerical tables for $W_{r}(\beta)$ are available (Vidal et al. 1971), which are accurate to 8 significant figures for the range $0 \leq \beta \leq 30$. Beyond this range, the known asymptotic approximation for $W_{r}(\beta)$ can be used, while a convenient interpolation on both $r$ and $\beta$ within the tables allows us to derive $W_{r}(\beta)$ in the conditions of densities $N_{\mathrm{e}}$ and temperatures $T$ of the experiments of Gauthier et al. (1981) and Bücher et al. (1995).

Hooper's approach is not applicable for the treatment of extremely dense plasmas (solar cores), i.e. in the limit of extremely strong coupling parameter $(\gamma \gg 10)$ : it has been primarily concerned with less dense plasmas, such as those produced in the laboratory.

\subsubsection{APEX distribution and analytic fitting formula}

The first theory able to provide reliable numerical results for the microfield distributions $W_{r}(\beta)$ in both weakly and strongly coupled plasmas proved to be the adjustable-parameter exponential approximation (APEX, Iglesias et al. 1983; Iglesias \& Lebowitz 1984; Iglesias 2000). This method can be derived from a renormalized cluster expansion that maximizes the independent-particle contribution relative to the BarangerMozer series (Iglesias et al. 2000). It is based on a formalism that expresses the Fourier transform of $W_{r}(\beta)$ in terms of a special term-distribution function containing a free parameter which is then fixed to give the exact second moment of $W_{r}(\beta)$ (Iglesias et al. 1983; Iglesias \& Lebowitz 1984; Iglesias 2000). The numerical results obtained from this scheme agree well with computed simulations for strongly coupled one-component plasmas.

However, the calculation of the different integrals involved in this method needs a considerable effort, especially in the case of correlated plasma. Thus, assuming that our system is isotropic, the microfield distribution $W_{r}(\beta)$ may be obtained by 
elementary differentiation of the cumulative probability distribution (CPD) $Q(\beta)$ which satisfies the relation

$Q(\beta)=\int_{0}^{\beta} W\left(\beta^{\prime}\right) \mathrm{d} \beta^{\prime}$

where $Q(\beta) . \mathrm{d} \beta$ denotes the probability of finding a normalized electric field $\beta$, at a singly charged or neutral point, due to a collection of $N$-charged particles moving in a uniform neutral background and contained in a volume $V$. The CPD function calculated by the APEX method for a neutral point in a correlated plasma can be approximated by the formula (Potekhin et al. 2002)

$Q(\beta)=\frac{a_{0} \beta^{3}-2 \beta^{9 / 2}+\beta^{6}}{a_{1}+a_{2} \beta+a_{3} \beta^{2}+a_{4} \beta^{3}-\beta^{9 / 2}+\beta^{6}}$,

where the fitting parameters $a_{i}$ can be approximated as functions of the coupling parameter $\gamma$ and the screening parameter $s$ defined as (Potekhin et al. 2002)

$s=R_{\mathrm{c}} k_{\mathrm{s}}$.

The analytic fitting formula of Eq. (42) has been checked for the range of the plasma parameters, i.e., at $0 \leq \Gamma \leq 100$ and $0 \leq s \leq 3$ (Potekhin et al. 2002). At $\Gamma=0$, the differentiation of the CPD function reproduces the Holtsmark data at any $\beta$ with a maximum fractional error of $0.24 \%$ (Potekhin et al. 2002). Therefore, it is suitable for our application.

In the linear first-order perturbation approximation, the effective electron-screening wave number $k_{\mathrm{s}}$ is related to the Fermi-Dirac integral $F_{n}(\chi)$ of order $-1 / 2$ (Potekhin et al. 2002)

$k_{\mathrm{s}}=C_{1} F_{-1 / 2}(\chi)$,

$F_{n}(\chi)=\int_{0}^{\infty} \frac{t^{n} \mathrm{~d} t}{\mathrm{e}^{t-\chi}-1}, \quad n>-1$,

$C_{1}=\frac{e}{\pi \hbar^{3}}\left(2 m_{\mathrm{e}}\right)^{3 / 2}\left(k_{\mathrm{B}} T\right)^{1 / 2}$.

The electronic chemical potential $\chi=\mu / k_{\mathrm{B}} T$ is determined from the inverse function $X_{n}(f)$ of the complete Fermi-Dirac integral of order $1 / 2$ (Potekhin et al. 2002)

$\chi=X_{1 / 2}\left(C_{2}\right)$,

$C_{2}=\frac{\pi^{2} \hbar^{3}}{\sqrt{2}}\left(m_{\mathrm{e}} k_{\mathrm{B}} T\right)^{-3 / 2} N_{\mathrm{e}}$.

The solutions of Eq. (45) and Eq. (47) may be determined by using accurate Padé approximations for the complete Fermi-Dirac integral $F_{n}(\chi)$ and its corresponding inverse function $X_{n}(f)$ (Antia 1993)

$$
\begin{aligned}
& F_{n}(\chi) \approx \begin{cases}e^{\chi} R_{m_{1} k_{1}}^{1}\left(e^{\chi}\right) & \chi<2 \\
\chi^{n+1} R_{m_{2} k_{2}}^{2}\left(\chi^{-2}\right) \quad \chi \geq 2,\end{cases} \\
& X_{n}(f) \approx \begin{cases}\operatorname{Ln}\left[f R_{m_{1} k_{1}}^{1}(f)\right] & \chi<4 \\
f^{1 /(n+1)} R_{m_{2} k_{2}}^{2}\left(f^{-1 /(n+1)}\right) & \chi \geq 4,\end{cases} \\
& R_{m_{1} k_{1}}^{1}(\chi)=\sum_{i=1}^{m_{1}} \widehat{a_{i}} \chi^{i} / \sum_{i=1}^{k_{1}} \widehat{b_{i}} \chi^{i},
\end{aligned}
$$

$$
R_{m_{2} k_{2}}^{1}(\chi)=\sum_{i=1}^{m_{2}} \widehat{c_{i}} \chi^{i} / \sum_{i=1}^{k_{2}} \widehat{d}_{i} \chi^{i}
$$

where the values of $m_{1}, k_{1}, m_{2}, k_{2}, \widehat{a_{i}}, \widehat{b_{i}}, \widehat{c_{i}}$ and $\widehat{d}_{i}$ are given in Antia (1993). These rational function minima approximations are used to efficiently compute the values of the integrals $F_{n}(\chi)$ and $X_{n}(f)$ with maximum relative errors $\approx 10^{-12}$ and $\approx 10^{-8}$, respectively.

\section{Calculation of the spectral width}

Numerical results are only given in the present paper for the HeI $6678 \AA\left(2^{1} \mathrm{P}^{\circ}-3^{1} \mathrm{D}\right)$ and $5876 \AA\left(2^{3} \mathrm{P}^{\circ}-3^{3} \mathrm{D}\right)$ lines, but the method can be readily extended to a wide class of spectral lines of other atoms.

To evaluate the total width $W_{\text {tot }}$ we must consider both electron and ion perturbing effects. The influence of electrons and ions can be treated separately due to the difference in mass and mobility. If the electron density is not very high, electron collisions are treated by the impact theory which takes into account deviations from adiabaticity. The corresponding profiles are, therefore, of dispersion (Lorentz-Weisskopf) type. For the ion effects, the adiabatic and in most cases the quasistatic approximation must be used, due to the high density of the experiment.

Several numerical modifications have been made in the original Sahal-Bréchot computer code in order to introduce the new collision functions and compute the different contributions to the width in the case of a non-ideal plasma.

The input parameters of the code are the atomic data relative to the considered transition, the density $N_{\mathrm{e}}$ of the plasma and its temperature $T$. In the present paper, energy levels and oscillator strength for the considered lines have been taken from TOPbase (Cunto et al. 1993; Zeippen 1995; The Opacity Project Team 1995). As TOPbase does not provide mean radii and mean square radii, we have calculated them within the hydrogenic approximation by using the effective quantum numbers $n_{i}^{*}$ obtained from the values of the energy levels.

\subsection{Impact electronic contribution}

The impact electronic total width $W_{\mathrm{e}}$ contains three contributions, i.e.: the elastic part and the two inelastic parts relative to the initial $i$ and final $f$ states. The inelastic contributions have been calculated taking into account the new collision functions (Ben Chaouacha et al. 2004, 2005).

Three main steps are executed to compute the impact electronic total width $W_{\mathrm{e}}$. First, the collision functions relative to the transition probability $A(z)$ and the cross section $a(z)$ are calculated for the three interaction potentials. Then, the $A(z)$ function is used to calculate the sum $\sum_{j \neq i} P_{i j}(\rho, v)$. In the same way, the $a(z)$ function is used to calculate the $\operatorname{sum} \sum_{j \neq i} \sigma_{i j}(v)$.

The perturbation theory used for the derivation of the $S$-matrix leads to a divergence in the integration over the impact parameter: a lower cut-off is thus required. For high densities or for very small energy differences, an upper cut-off is also used, in order to take into account the shielding. The symmetrization procedures and the choice of the lower cut-off to enforce the unitarity of the $S$-matrix have been widely discussed in Feautrier (1968) and Sahal-Bréchot (1969a,b).

By applying the same procedure to both the initial $\mathrm{i}\left(n_{\mathrm{i}} l_{\mathrm{i}} L_{\mathrm{i}} S_{\mathrm{i}} J_{\mathrm{i}}\right)$ and the final $\mathrm{f}\left(n_{\mathrm{f}} l_{\mathrm{f}} L_{\mathrm{f}} S_{\mathrm{f}} J_{\mathrm{f}}\right)$ states, the total cross section $\sigma_{t}(v)$ will be calculated using Eq. (19). 
The integral in Eq. (18) is calculated numerically using the trapezoid method with an exponential step (Feautrier 1968; Sahal-Bréchot 1969a,b).

\subsection{Quasistatic ionic contribution}

The quasistatic ionic contribution $W_{\text {quas }}$ can be estimated using the approach developed by Griem et al. (1962) and Griem (1974), due to its simple applicability to different kinds of perturbing ions.

For most of the isolated lines, it is convenient to use the impact electron width as the unit of frequency detuning from the line shifted by electron impacts, i.e., to introduce as a reduced variable the frequency shift $x$ (Griem et al. 1962; Griem 1974)

$x=\frac{\omega-\omega_{0}-d}{W_{\mathrm{e}}}$,

where $\omega_{0}$ and $\omega$ are the angular frequencies of the unperturbed and the perturbed lines, $W_{\mathrm{e}}$ is the impact electronic total width in angular frequency, and $d$ is the impact electronic shift also in angular frequency unit. The reduced profile of allowed components is expressed as (Griem et al. 1962; Griem 1974)

$j_{A, r}(x)=\frac{1}{\pi} \int_{0}^{\infty} \frac{W_{r}(\beta) \mathrm{d} \beta}{1+\left(x-A^{4 / 3} \beta^{2}\right)^{2}}$.

The quasistatic quadratic ion broadening parameter $A$, denoted by $\alpha$ in Griem et al. (1962) and Griem (1964), is a measure of the relative importance of ion broadening. It represents the quasistatic ionic correction without the Debye cut-off, while $r$ is the quasistatic ionic correction due to the Debye cut-off; its expression can be written as (see e.g. Ben Nessib et al. 1996)

$A=\left(\frac{e F_{0}^{2}}{\hbar W_{\mathrm{e}}}\left|\alpha_{i}-\alpha_{f}\right|\right)^{3 / 4}$,

where the atomic polarizability $\alpha_{i}$ of the level $i$ (resp. $f$ ) is given by

$\alpha_{i}=4 a_{0}^{3} \sum_{j \neq i} f_{i j}\left(\frac{I_{\mathrm{H}}}{\Delta E_{i j}}\right)^{2}$,

where $a_{0}$ is the Bohr radius and $I_{\mathrm{H}}$ the ionization energy of hydrogen. It can be seen from this definition that its magnitude is determined by the magnitude of the oscillator strengths $f_{i j}$ and the energy difference $\Delta E_{i j}$ between the levels $j$ and $i$. Since $W_{\mathrm{e}}$ is proportional to $N_{\mathrm{e}}$, the parameter $A$ is only proportional to $N_{\mathrm{e}}^{1 / 4}$.

Contrary to the $A$-value of Griem who used a simplified Coulomb approximation for the atomic structure (Griem et al. 1962; Griem 1974), the quasistatic ion broadening parameter $A$ is computed with the TOPbase sophisticated atomic structure as in Ben Nessib et al. (1996).

The quasistatic approximation will be valid provided the frequencies characterizing the ion field, namely $v_{\text {ion }} / R_{\mathrm{c}}$, which is of the order of the inverse of the duration of an interaction $\left[v_{\text {ion }}\right.$ being a typical ion velocity, $R_{\mathrm{c}}$ is the mean ion-ion separation], are considerably smaller than $W_{\mathrm{e}}$, which essentially determines the width of the profile (Griem 1962). If the parameter

$\sigma=W_{\mathrm{e}} R_{\mathrm{c}} / v_{\text {ion }}=W_{\mathrm{e}}\left(4 \pi N_{\mathrm{e}} / 3\right)^{-1 / 3} / v_{\text {ion }}$

becomes of order 1 or smaller, the time dependence of the ion field can no longer be neglected (Griem 1974).
Equation (54) is only applicable if the quasistatic approximation holds for ion broadening, which is justified for $\sigma>1$. In addition, it cannot be expected to be accurate for $r \approx 1$ or larger, because the Debye theory is no longer valid and the cluster integral expansion should be carried further. This restriction on $r$ is not serious because, for densities corresponding to larger values of this parameter, spectral lines are usually too broad to be observable. However, the opposite extreme, $r \gg 1$, rarely occurs in plasma spectroscopy, and a discussion of calculations for such situations may therefore be omitted (Griem 1962).

\subsection{Total width}

$\Delta x$ being the Full Width at Half Maximum (FWHM) in reduced units, obtained from the computed and tabulated $j_{A, r}(x)$, the total width $W_{\text {tot }}$ of the line in frequency units is

$W_{\text {tot }}=W_{\mathrm{e}} \Delta x$.

Moreover, an approximate formula for the total width $W_{\text {tot }}^{\mathrm{G}}$ of the complete profile can be obtained (Griem 1962, 1974)

$W_{\text {tot }}^{\mathrm{G}} \approx[1+1.75 A(1-0.75 r)] W_{\mathrm{e}}$.

The formula of Eq. (59) is adapted only for an ideal plasma studied with the Coulomb Debye model. It is reasonably accurate in view of the uncertainties introduced by the various other approximations, as long as $A \leq 0.5$ and $r \leq 0.8$ (Griem 1974). For higher values of $A$, Eq. (58) must be used.

\section{Results and discussion}

To validate our theoretical approach, the different contributions to the width are calculated for the HeI $6678 \AA\left(2{ }^{1} \mathrm{P}^{\circ}-3{ }^{1} \mathrm{D}\right)$ and $5876 \AA\left(2^{3} \mathrm{P}^{\circ}-3^{3} \mathrm{D}\right)$ lines, by considering the same conditions of densities $N_{\mathrm{e}}$ and temperatures $T$ as those of the experiments of Gauthier et al. (1981) and Bücher et al. (1995).

The impact approximation criterion is well satisfied for electronic collisions. In a first step, the impact electronic total width was calculated in the case of the Coulomb-Debye potential, i.e. $W_{\mathrm{e}}^{\mathrm{CD}}$, with an upper cutoff at $R_{\mathrm{D}}$. By calculating the relative contribution of the strong collisions to the impact total width, the validity conditions of the perturbation theory can also be checked.

Table 2 shows that the electronic strong collision contribution $W_{\mathrm{s}, \mathrm{e}}^{\mathrm{CD}}$ does not exceed $30 \%$ of the impact electronic total width $W_{\mathrm{e}}^{\mathrm{CD}}$. Consequently, the perturbation theory approximation is well satisfied for collisions with electrons for these lines. About $90 \%$ to $95 \%$ of the inelastic electronic part is related to the upper level (Table 2).

Figure 1 compares the impact electronic total width $W_{\mathrm{e}}^{\mathrm{CD}}$, the elastic contribution $W_{\mathrm{e}, \mathrm{el}}^{\mathrm{CD}}$ and the inelastic contribution $W_{\mathrm{e}, \text { inel }}^{\mathrm{CD}}$. It can be noted that for the two lines the inelastic collisions give the largest contribution. The two contributions $W_{\mathrm{e}, \text { inel }}^{\mathrm{CD}}$ and $W_{\mathrm{e}, \mathrm{el}}^{\mathrm{CD}}$ present almost the same behavior and increase with the electronic density $N_{\mathrm{e}}$. The elastic term $W_{\mathrm{e}, \mathrm{el}}^{\mathrm{CD}}$ is relatively low and does not exceed $15 \%$ of the impact electronic total width $W_{\mathrm{e}}^{\mathrm{CD}}$. The inelastic contributions due to weak collisions are dominant $(70 \%)$, because the excited states of the HeI are very close together (Sahal-Bréchot 1969a,b).

In a second step, we have calculated the inelastic electronic contribution in the case of the Cut-off and Ion Sphere potentials, with an upper cut-off at $R_{\mathrm{c}}$. Figure 2 compares the evolution of the inelastic electronic contributions $W_{\mathrm{e}, \text { inel }}^{\mathrm{CD}}, W_{\mathrm{e}, \text { inel }}^{\mathrm{C}}$ and $W_{\mathrm{e}, \text { inel }}^{\mathrm{IS}}$ for the considered lines. The two inelastic widths $W_{\mathrm{e}, \text { inel }}^{\mathrm{CD}}$ and $W_{\mathrm{e}, \text { inel }}^{\mathrm{C}}$ 
Table 2. Different contributions to the width calculated for the considered HeI transitions. $W^{\text {exp }}$ : experimental width $-W_{\mathrm{e}}^{\mathrm{CD}}$ : impact electronic total width $-W_{\mathrm{s}, \mathrm{e}}^{\mathrm{CD}}$ : width due to the strong electronic collisions - $W_{\mathrm{e}, \mathrm{el}}^{\mathrm{CD}}$ : impact elastic electronic contribution $-W_{\mathrm{e}, \text { inel }}^{\mathrm{CD}, \mathrm{L}}$ : impact inelastic electronic contribution of the lower level $-W_{\mathrm{e}, \text { inel }}^{\mathrm{CD}, \mathrm{U}}$ : impact inelastic electronic contribution of the upper level.

\begin{tabular}{|c|c|c|c|c|c|c|c|}
\hline Transition & $\begin{array}{c}T \\
\left(10^{4} \mathrm{~K}\right) \\
\end{array}$ & $\begin{array}{c}N_{\mathrm{e}} \\
\left(10^{17} \mathrm{~cm}^{-3}\right)\end{array}$ & $\begin{array}{l}W^{\exp } \\
(\AA)\end{array}$ & $\begin{array}{l}W_{\mathrm{e}}^{\mathrm{CD}} \\
(\AA)\end{array}$ & $\begin{array}{l}W_{\mathrm{s}, \mathrm{e}}^{\mathrm{CD}} \\
(\AA) \\
\end{array}$ & $\begin{array}{l}W_{\mathrm{e}, \mathrm{el}}^{\mathrm{CD}} \\
(\AA) \\
\end{array}$ & $\begin{array}{c}\frac{W_{\mathrm{e}, \text { inel }}^{\mathrm{CD}, \mathrm{L}}}{W_{\mathrm{e}, \text { inel }}^{\mathrm{CD}}} \\
(\%)\end{array}$ \\
\hline$\left(2^{1} \mathrm{P}^{\circ}-3^{1} \mathrm{D}\right)$ & 1.35 & 2.0 & 8.6 & 11.4 & 2.8 & 1.0 & 5.6 \\
\hline \multirow[t]{12}{*}{$6678 \AA$} & 1.78 & 3.3 & 12.9 & 17.2 & 4.2 & 1.7 & 6.5 \\
\hline & 1.86 & 4.9 & 15.5 & 24.2 & 6.3 & 2.5 & 7.1 \\
\hline & 1.93 & 6.2 & 19.9 & 29.4 & 7.8 & 3.1 & 7.5 \\
\hline & 2.01 & 6.9 & 20.7 & 32.2 & 8.6 & 3.4 & 7.7 \\
\hline & 2.13 & 8.4 & 25.0 & 37.7 & 10.3 & 4.2 & 8.1 \\
\hline & 2.09 & 8.9 & 25.9 & 39.7 & 11.0 & 4.4 & 8.2 \\
\hline & 2.28 & 10.0 & 28.5 & 43.6 & 12.1 & 4.9 & 8.6 \\
\hline & 2.32 & 10.7 & 30.2 & 46.2 & 12.9 & 5.3 & 8.8 \\
\hline & 2.40 & 11.8 & 31.9 & 50.0 & 14.1 & 5.8 & 9.0 \\
\hline & 2.44 & 13.2 & 35.4 & 54.7 & 15.6 & 6.5 & 9.3 \\
\hline & 2.59 & 13.7 & 36.3 & 56.6 & 16.0 & 6.8 & 9.5 \\
\hline & 2.55 & 14.9 & 40.6 & 60.5 & 17.5 & 7.3 & 9.7 \\
\hline$\left(2^{3} \mathrm{P}^{\circ}-3^{3} \mathrm{D}\right)$ & 4.46 & 4.90 & 17.5 & 13.8 & 4.0 & 1.9 & 9.0 \\
\hline \multirow[t]{4}{*}{$5876 \AA$} & 5.20 & 10.2 & 36.7 & 28.0 & 8.1 & 3.9 & 10.2 \\
\hline & 5.21 & 12.0 & 43.2 & 32.6 & 9.6 & 4.6 & 10.4 \\
\hline & 5.60 & 18.4 & 66.0 & 48.9 & 14.6 & 7.1 & 11.2 \\
\hline & 5.35 & 24.9 & 89.2 & 65.1 & 19.5 & 9.4 & 12.2 \\
\hline
\end{tabular}

increase with the plasma density $N_{\mathrm{e}}$, and their shape is almost the same. For the $6678 \AA$ line, the Ion Sphere contribution $W_{\mathrm{e}, \text { inel }}^{\mathrm{IS}}$ decreases when $N_{\mathrm{e}}$ exceeds $10^{16} \mathrm{~cm}^{-3}$. The three contributions converge at weak plasma densities $\left(N_{\mathrm{e}} \approx 10^{15} \mathrm{~cm}^{-3}\right)$ since the effects of the corrective terms become negligible, i.e., the plasma may be considered effectively as ideal.

In the case of a non-ideal plasma $\left(N_{\mathrm{e}}>10^{15} \mathrm{~cm}^{-3}\right)$, the two correlated inelastic contributions $W_{\mathrm{e}, \text { inel }}^{\mathrm{C}}$ and $W_{\mathrm{e}, \text { inel }}^{\mathrm{IS}}$ are smaller than the Coulomb-Debye one, following the general tendency of the corresponding collision functions $A(z)$ and $a(z)$ (Ben Chaouacha et al. 2004, 2005). The contribution relative to the Ion Sphere model $W_{\mathrm{e}, \text { inel }}^{\mathrm{IS}}$ is the lowest; in parallel, the difference between the two correlated terms $W_{\mathrm{e} \text {,inel }}^{\mathrm{C}}$ and $W_{\mathrm{e} \text {,inel }}^{\mathrm{IS}}$ tends to increase as the electronic density $N_{\mathrm{e}}$ increases. However, if the density $N_{\mathrm{e}}$ exceeds the value $\left(N_{\mathrm{e}} \geq 10^{18} \mathrm{~cm}^{-3}\right)$, this tendency is expected to no longer be systematic: the semi-classical approach and the Ion Sphere model are expected to be not well adapted to describe a plasma in such extreme conditions.

We have calculated the elastic contribution only with the Coulomb-Debye model $\left(W_{\mathrm{e}, \mathrm{Cl}}^{\mathrm{CD}}\right)$. The validity of this assumption is justified by the fact that the elastic cross sections are mainly due to strong collisions: Table 3 shows that the ratio of the impact elastic electronic contribution to the electronic density $\frac{W_{\mathrm{e}, \mathrm{el}}^{\mathrm{CD}}}{N_{\mathrm{e}}}$ is quite constant, since the difference does not exceed $1 \%$ for the two lines. Therefore, the upper cut-off at $R_{\mathrm{D}}$ has a negligible effect for the elastic contribution, even at high electronic densities. In addition, still concerning elastic collisions, the contribution of the quadratic $r_{\mathrm{p}}^{-4}$-potential which is short-range is dominant compared to the quadrupolar one. Hence, the very long-range collisions are not important, and the results for the elastic contribution should not be very different in the case of
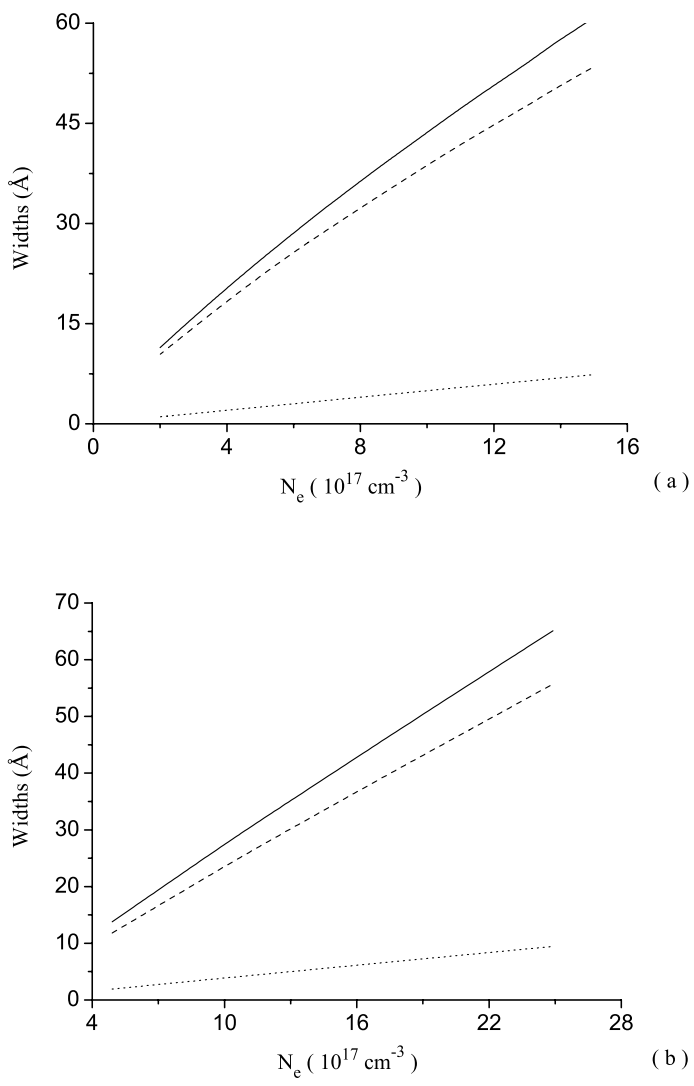

Fig. 1. Different contributions to the impact electronic total width $W_{\mathrm{e}}^{\mathrm{CD}}$ calculated for the considered HeI transitions. Straight line: impact electronic total width $W_{\mathrm{e}}^{\mathrm{CD}}$; Dashed line: inelastic electronic contribution $W_{\mathrm{e}, \text { inel }}^{\mathrm{CD}}$; Dotted line: elastic electronic contribution $W_{\mathrm{e}, \mathrm{el}}^{\mathrm{CD}}$. [a): $6678 \AA$ $\left.\left.\left(2^{1} \mathrm{P}^{\circ}-3^{1} \mathrm{D}\right), \mathbf{b}\right): 5876 \AA\left(2^{3} \mathrm{P}^{\circ}-3^{3} \mathrm{D}\right)\right]$.

the Coulomb-Debye, Cut-off and Ion Sphere models. However, this might be improved in a further paper.

Table 4 lists the values of the Debye shielding parameter $r$ and the three quasistatic quadratic ion broadening parameters $A^{\mathrm{CD}}, A^{\mathrm{C}}$ and $A^{\mathrm{IS}}$ for the studied lines. They are obtained from Eq. (55) by using the associated impact electronic total widths $W_{\mathrm{e}}^{\mathrm{CD}}, W_{\mathrm{e}}^{\mathrm{C}}$ and $W_{\mathrm{e}}^{\mathrm{IS}}$. For the $6678 \AA$ line, $r \approx 0.6$ and that $A^{\mathrm{CD}}$ is slightly higher than the upper limit of validity $(0.5)$. Hence, for the Coulomb Debye model, the total width $W_{\text {tot }}$ has been estimated using the approximated formula of Eq. (59). For both the Cut-off and the Ion Sphere models, the formulae given by Eq. (58) and Eq. (54) have been used, since $A^{\mathrm{C}}$ and $A^{\mathrm{IS}}$ are much greater than unity. For the $5876 \AA$ line, $r \approx 0.4$ and only $A^{\mathrm{IS}}$ is slightly higher than the upper limit of validity $(0.5)$.

By considering the values of $r, A^{\mathrm{CD}}, A^{\mathrm{C}}$ and $A^{\mathrm{IS}}$ tabulated in Table 4 , the complete reduced Stark profiles $j_{A, r}(x)$ of isolated lines have been evaluated numerically. In a first step, we checked our code by verifying that our numerical procedure reproduces the same data tabulated in Griem (1974). Then, the $j_{A, r}(x)$ profiles were analyzed to determine their Full Width at Half Maximum (FWHM) $\Delta x$.

The three total widths $W_{\text {tot }}^{\mathrm{CD}}, W_{\text {tot }}^{\mathrm{C}}$ and $W_{\text {tot }}^{\mathrm{IS}}$ of the considered lines obtained from the expression of Eq. (58), by considering micro-field distributions $W_{r}(\beta)$ derived with the different numerical methods presented above are compared in Figs. 3 and 4 to the experimental widths $W^{\exp }$ of Gauthier et al. (1981) and 

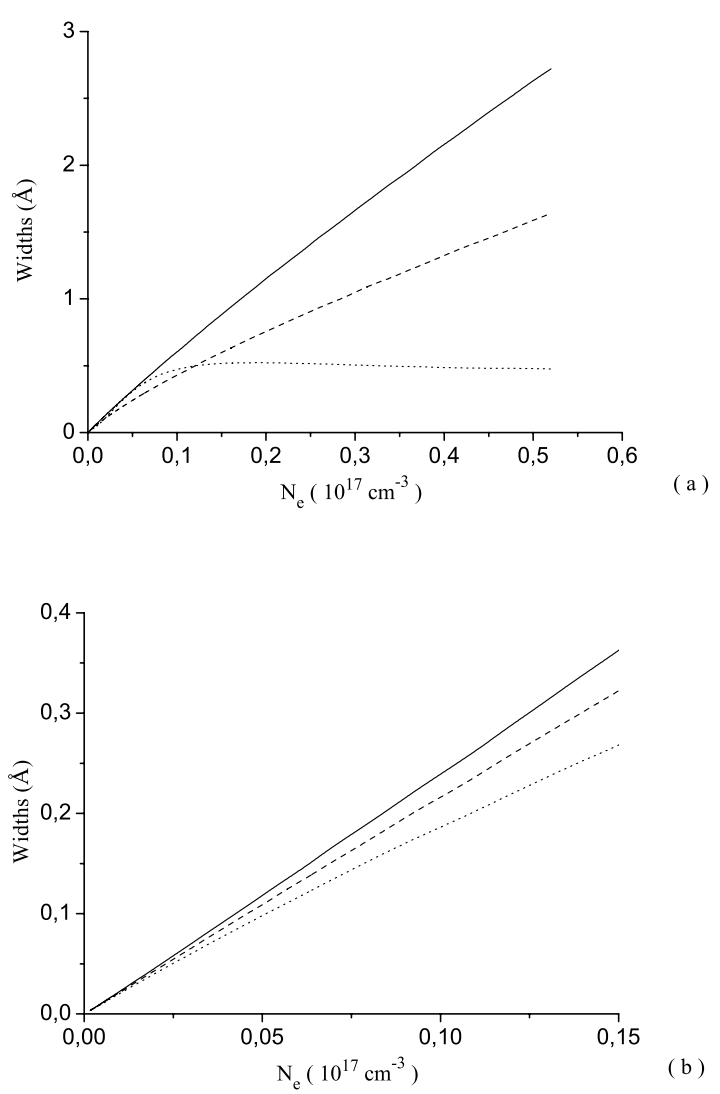

( b )

Fig. 2. Impact inelastic electronic widths $W_{\mathrm{e}, \text { inel }}^{\mathrm{CD}}, W_{\mathrm{e}, \text { inel }}^{\mathrm{C}}$, and $W_{\mathrm{e}, \text { inel }}^{\mathrm{IS}}$ calculated for the considered HeI transitions, by considering a small nonideality factor $(\gamma=0.03)$. Straight line: $W_{\mathrm{e}, \text { inel }}^{\mathrm{CD}}$; Dashed line: $W_{\mathrm{e}, \mathrm{inel}}^{\mathrm{C}}$; Dotted line: $W_{\mathrm{e}, \text { inel }}^{\mathrm{IS}}$. [a): $\left.\left.6678 \AA\left(2^{1} \mathrm{P}^{\circ}-3^{1} \mathrm{D}\right), \mathbf{b}\right): 5876 \AA\left(2^{3} \mathrm{P}^{\circ}-3^{3} \mathrm{D}\right)\right]$.

Table 3. Ratio of the impact elastic electronic contribution to the electronic density $\frac{W_{\mathrm{e}, \mathrm{el}}^{\mathrm{CD}}}{N_{\mathrm{e}}}\left(10^{-18} \AA \mathrm{cm}^{3}\right)$ calculated for the considered HeI transitions.

\begin{tabular}{cccccc}
\hline \hline Transition & $\begin{array}{c}N_{\mathrm{e}}\left(10^{17} \mathrm{~cm}^{-3}\right) \\
T\left(10^{4} \mathrm{~K}\right)\end{array}$ & 0.1 & 1.0 & 10.0 & 16.0 \\
\hline$\left(2^{1} \mathrm{P}^{\circ}-3^{1} \mathrm{D}\right)$ & 1.0 & 5.57 & 5.56 & 5.42 & 5.35 \\
$6678 \AA$ & 1.5 & 5.15 & 5.14 & 5.09 & 5.06 \\
& 2.0 & 5.00 & 5.00 & 4.97 & 4.96 \\
& 2.5 & 4.95 & 4.94 & 4.93 & 4.92 \\
$\left(2^{3} \mathrm{P}^{\circ}-3^{3} \mathrm{D}\right)$ & 1.0 & 6.25 & 6.25 & 6.22 & 6.19 \\
$5876 \AA$ & 1.5 & 5.41 & 5.41 & 5.40 & 5.39 \\
& 2.0 & 4.88 & 4.88 & 4.87 & 4.86 \\
& 2.5 & 4.57 & 4.57 & 4.56 & 4.56 \\
\hline
\end{tabular}

Bücher et al. (1995). Unfortunately, these experiments correspond to relatively weak values of the non-ideality factor $(\gamma<$ 0.3 ). It is generally difficult to provide experimental data at the high electronic densities $N_{\mathrm{e}}$ required by the Ion Sphere model.

Figure 3 shows that for the $6678 \AA$ line the different total widths $W_{\text {tot }}^{\mathrm{CD}}, W_{\text {tot }}^{\mathrm{C}}$ and $W_{\text {tot }}^{\mathrm{IS}}$ increase with the plasma density $N_{\mathrm{e}}$ and are more important with a micro-field distribution $W_{r}(\beta)$ derived by the AFF method (Potekhin et al. 2002). The Coulomb Debye model overestimates the experimental data for a micro-field distribution $W_{r}(\beta)$ derived either by Baranger \& Mozer's method (Baranger \& Mozer 1959; Mozer \& Baranger 1960 ) or by Hooper's method (Hooper 1966, 1968a,b), $W_{\text {tot }}^{\mathrm{CD}}$ exceeds $310 \%$ of $W^{\text {exp }}$, and this difference is close to $350 \%$ for the APEX method. This confirms the importance of taking into account the correlations in a non-ideal plasma. The Cut-off model also disagrees with the experimental data: $W_{\text {tot }}^{\mathrm{C}}$ is greater than the experimental width $W^{\text {exp }}$ and a difference close to $300 \%$ is typically obtained at high electron densities $N_{\mathrm{e}}$ for the APEX method. Therefore, this correlated model cannot reproduce all the physical considerations. The Ion Sphere model presents a difference with the experiment, but this difference tends to be the closest one for the electron density range $\left(4 \times 10^{17} \mathrm{~cm}^{-3}<N_{\mathrm{e}}<9 \times 10^{17} \mathrm{~cm}^{-3}\right)$ and with a microfield distribution $W_{r}(\beta)$ derived by Hooper's method. However, $W_{\text {tot }}^{\mathrm{IS}}$ does not exceed $63 \%$ of $W^{\text {exp }}$ at low electron densities $N_{\mathrm{e}}$, while a difference close to $54 \%$ is obtained at high electron densities $N_{\mathrm{e}}$.

For the $5876 \AA$ line, the correlated total widths $W_{\text {tot }}^{\mathrm{C}}$ and $W_{\text {tot }}^{\mathrm{IS}}$ are lower than $W^{\exp }$ (Fig. 4). A difference close to $4 \%$ is obtained with the Coulomb Debye model and with a microfield distribution $W_{r}(\beta)$ derived by Hooper's method. Hence, this discussion shows that our theoretical approach would require new refinements in order to be in perfect agreement with the experiment.

Table 5 shows that the Coulomb-Debye total width $W_{\text {tot }}^{\mathrm{CD}}$ calculated from the associated Full Width at Half Maximum (FWHM) $\Delta x^{\mathrm{CD}}$ using Eq. (58) is in good agreement with the approximated total width $W_{\text {tot }}^{\mathrm{G}}$ obtained from Eq. (59), by considering the quasistatic quadratic ion broadening parameters $A^{\mathrm{CD}}$ and the Debye shielding parameter $r$ tabulated in Table 4: their difference does not exceed $3 \%$. However, the total width $W_{\mathrm{tot}}^{\mathrm{G}, *}$ obtained from the expression of Eq. (59) without taking into account the quasistatic ionic correction due to the Debye cut-off ( $r=0$ ) is greater than $W_{\text {tot }}^{\mathrm{G}}$ (up to $136 \%$, for the $6678 \AA$ line) (Table 5). Thus, $r$ cannot be neglected if the electronic density $N_{\mathrm{e}}$ is relatively high.

To generalize such result to the two correlated models, approximate formulae for the total width $W_{\text {tot,app }}$ similar to the expression of Eq. (59) are derived, by introducing two constants $a$ and $b$ so that

$W_{\text {tot,app }} \approx W_{\mathrm{e}}(a r+b A+1)$.

The parameters $a$ and $b$ can be readily obtained by a Least Square method using the following equations

$Y=a X+b$,

$X=r$

$Y=\frac{\left(\frac{W_{\mathrm{tot}}}{W_{\mathrm{e}}}-1\right)}{A}$,

where $W_{\text {tot }}$ is previously obtained using Eq. (58). The resulting formulae, in turn, are

$W_{\text {tot,app }}^{\mathrm{CD}} \approx W_{\mathrm{e}}^{\mathrm{CD}}\left(a^{\mathrm{CD}} r+b^{\mathrm{CD}} A^{\mathrm{CD}}+1\right)$,

$W_{\text {tot,app }}^{\mathrm{C}} \approx W_{\mathrm{e}}^{\mathrm{C}}\left(a^{\mathrm{C}} r+b^{\mathrm{C}} A^{\mathrm{C}}+1\right)$,

$W_{\text {tot,app }}^{\mathrm{IS}} \approx W_{\mathrm{e}}^{\mathrm{IS}}\left(a^{\mathrm{IS}} r+b^{\mathrm{IS}} A^{\mathrm{IS}}+1\right)$,

where the values of the associated parameters $\left(a^{\mathrm{CD}}, b^{\mathrm{CD}}\right),\left(a^{\mathrm{C}}\right.$, $\left.b^{\mathrm{C}}\right)$ and $\left(a^{\mathrm{IS}}, b^{\mathrm{IS}}\right)$ are tabulated in Table 6 . As shown in Table 5, the three total widths $W_{\text {tot,app }}^{\mathrm{CD}}, W_{\text {tot,app }}^{\mathrm{C}}$ and $W_{\text {tot,app }}^{\mathrm{IS}}$ are comparable to those calculated using Eq. (58). 
Table 4. Quasistatic quadratic ion broadening parameters $A^{\mathrm{CD}}, A^{\mathrm{C}}$ and $A^{\mathrm{IS}}$ calculated for the considered HeI transitions. $r=\frac{R_{\mathrm{c}}}{R_{\mathrm{D}}}$ : Debye shielding parameter $-W_{\mathrm{e}}^{\mathrm{CD}}, W_{\mathrm{e}}^{\mathrm{C}}$ and $W_{\mathrm{e}}^{\mathrm{IS}}$ : impact electronic total widths.

\begin{tabular}{cccccccccc}
\hline \hline Transition & $\begin{array}{c}T \\
\left(10^{4} \mathrm{~K}\right)\end{array}$ & $\begin{array}{c}N_{\mathrm{e}} \\
\left(10^{17} \mathrm{~cm}^{-3}\right)\end{array}$ & $r$ & $\begin{array}{c}W_{\mathrm{e}}^{\mathrm{CD}} \\
(\AA)\end{array}$ & $\begin{array}{c}W_{\mathrm{e}}^{\mathrm{C}} \\
(\AA)\end{array}$ & $\begin{array}{c}W_{\mathrm{e}}^{\mathrm{IS}} \\
(\AA)\end{array}$ & $A^{\mathrm{CD}}$ & $A^{\mathrm{C}}$ & $A^{\mathrm{IS}}$ \\
\hline$\left(2 \mathrm{P}^{\circ}-3^{1} \mathrm{D}\right)$ & 1.35 & 2.0 & 0.591 & 11.4 & 5.1 & 1.46 & 0.46 & 0.84 & 2.15 \\
$6678 \AA$ & 1.78 & 3.3 & 0.559 & 17.2 & 7.8 & 2.37 & 0.56 & 1.01 & 2.45 \\
& 1.86 & 4.9 & 0.586 & 24.2 & 10.6 & 3.47 & 0.64 & 1.20 & 2.77 \\
& 1.93 & 6.2 & 0.597 & 29.4 & 12.7 & 4.34 & 0.70 & 1.32 & 2.94 \\
& 2.01 & 6.9 & 0.596 & 32.2 & 13.9 & 4.84 & 0.73 & 1.37 & 3.03 \\
& 2.13 & 8.4 & 0.598 & 37.7 & 16.3 & 5.77 & 0.79 & 1.47 & 3.21 \\
& 2.09 & 8.9 & 0.610 & 39.7 & 17.1 & 6.09 & 0.81 & 1.52 & 3.29 \\
& 2.28 & 10.0 & 0.595 & 43.6 & 19.0 & 6.84 & 0.84 & 1.57 & 3.38 \\
& 2.32 & 10.7 & 0.597 & 46.2 & 20.2 & 7.28 & 0.87 & 1.61 & 3.46 \\
& 2.40 & 11.8 & 0.597 & 50.0 & 21.9 & 7.97 & 0.90 & 1.67 & 3.57 \\
& 2.44 & 13.2 & 0.603 & 54.7 & 24.0 & 8.86 & 0.94 & 1.74 & 3.67 \\
$\left(2^{3} \mathrm{P}^{\circ}-3^{3} \mathrm{D}\right)$ & 2.59 & 13.7 & 0.589 & 56.6 & 25.1 & 9.25 & 0.95 & 1.75 & 3.70 \\
$5876 \AA$ & 2.55 & 14.9 & 0.601 & 60.5 & 26.7 & 9.98 & 0.98 & 1.82 & 3.80 \\
& 4.46 & 4.9 & 0.378 & 13.8 & 9.2 & 5.42 & 0.19 & 0.26 & 0.39 \\
& 5.20 & 10.2 & 0.396 & 28.0 & 17.3 & 7.10 & 0.24 & 0.34 & 0.66 \\
& 5.21 & 12.0 & 0.406 & 32.6 & 19.7 & 7.71 & 0.25 & 0.36 & 0.73 \\
& 5.60 & 18.4 & 0.420 & 48.9 & 28.2 & 10.3 & 0.28 & 0.42 & 0.89 \\
& 6.35 & 24.9 & 0.415 & 65.1 & 36.8 & 13.5 & 0.30 & 0.47 & 0.99 \\
\hline
\end{tabular}

\section{Summary of the results and conclusion}

Ben Chaouacha et al. $(2004,2005)$ derived new semi-classical collision functions for both the transition probability and cross section by using the classical path approximation in the standard formalism of Stark impact broadening of spectral lines. These functions have been used in the present paper to compute the inelastic contribution $W_{\mathrm{e} \text {,inel }}$ to the electronic total width $W_{\mathrm{e}}$ in the case of a non-ideal plasma, by considering three different interaction potentials (Coulomb-Debye, Cut-off, and Ion Sphere). An upper cut-off at $R_{\mathrm{D}}$ for the Coulomb-Debye model (ideal case) and at $R_{\mathrm{c}}$ for the Cut-off and the Ion Sphere models has been used. To validate our theoretical approach, the numerical results have been calculated for the HeI $6678 \AA\left(2{ }^{1} \mathrm{P}^{\circ}-3^{1} \mathrm{D}\right)$ and $5876 \AA$ $\left(2^{3} \mathrm{P}^{\circ}-3^{3} \mathrm{D}\right)$ transitions, by considering the same conditions of densities $N_{\mathrm{e}}$ and temperatures $T$ as those of the experiments of Gauthier et al. (1981) and Bücher et al. (1995).

The lines corresponding to these transitions are well isolated and the plasma is weakly non-ideal for all temperatures and electronic densities of interest. For electronic collisions, the semiclassical perturbation theory is sufficient and the impact approximation is well satisfied.

The contribution of elastic electronic collisions $W_{\mathrm{e}, \mathrm{el}}$ calculated by considering the Coulomb Debye model is weak for these lines. Since the very long-range collisions are not important for this contribution, we consider that the corresponding results should not be very different in the case of the Cut-off and the Ion Sphere potentials.

The inelastic contribution $W_{\text {e,inel }}$ depends both on the choice of the non-ideality $\gamma$-value and the interaction potential model. The three impact inelastic electronic widths $W_{\mathrm{e}, \text { inel }}^{\mathrm{CD}}, W_{\mathrm{e}, \text { inel }}^{\mathrm{C}}$ and $W_{\mathrm{e} \text { inel }}^{\mathrm{IS}}$ become of the same order of magnitude at weak plasma densities $\left(N_{\mathrm{e}} \approx 10^{15} \mathrm{~cm}^{-3}\right)$. The two correlated widths are lower than the ideal one, which is in agreement with the general behavior of the associated collision functions.

The ion effects on such a line are as important as the electron ones. The impact approximation fails for ionic collisions, due to the relatively high densities prevailing in these experimental conditions. The interactions with the ions may be treated within the quasistatic approximation. In that case, atom-ion interaction is quadratic. Therefore, the quasistatic ionic contribution $W_{\text {quas }}$ is dominated by the polarization (or quadratic) $r_{\mathrm{p}}^{-4}$-interaction.

To consider both the electron and the ion effects, the total width $W_{\text {tot }}$ is obtained from the Full Width at Half Maximum (FWHM) $\Delta x$ deduced from the complete reduced Stark profile $j_{A, r}(x)$ of isolated lines. The Debye shielding parameter $r$ and the quasistatic quadratic ion broadening parameter $A$ are relevant to numerically evaluate such profiles. For comparison, the calculations of the associated microfield distributions $W_{r}(\beta)$ are performed using three different numerical methods.

The computed total widths $W_{\text {tot }}^{\mathrm{CD}}, W_{\text {tot }}^{\mathrm{C}}$ and $W_{\text {tot }}^{\mathrm{IS}}$ relative to the considered lines are compared to the corresponding experimental widths $W^{\exp }$ (Gauthier et al. 1981; Bücher et al. 1995). For the $5876 \AA$ Aine, the Coulomb Debye width $W_{\text {tot }}^{\mathrm{CD}}$ is comparable to $W^{\exp }$. For the $6678 \AA$ line, the Ion Sphere model gives the best results with a microfield distribution $W_{r}(\beta)$ derived by Hooper's method (1966, 1968a,b): such a numerical method is sufficient for use in our case. A difference close to $50 \%$ is expected at both low and high electron densities $N_{\mathrm{e}}$. Such a discrepancy may be due to different reasons, which are discussed in the following.

It is impossible to have a perfect agreement between the calculated widths and the experimental data only by improving the calculation of the collision functions: our model only improves the inelastic electronic contribution $W_{\mathrm{e} \text {,inel }}$ to the impact electronic total width $W_{\mathrm{e}}$; it takes into account the quasistatic ionic contribution.

Since a real plasma is intrinsically dynamic and complex, it may be studied by considering the collective effects rather than the binary ones: such effects cannot be described by a simple corrective term in the interaction potential expression.

The calculation of the excitation cross section needs the introduction of a minimum cut-off radius which may eliminate certain significant terms in the numerical integration procedure.

A semi-classical treatment for electron collisions neglects the emitter-perturber exchange, which is mainly due to the strong collisions. Hence, when strong collisions are abundant, quantum effects must be considered. 

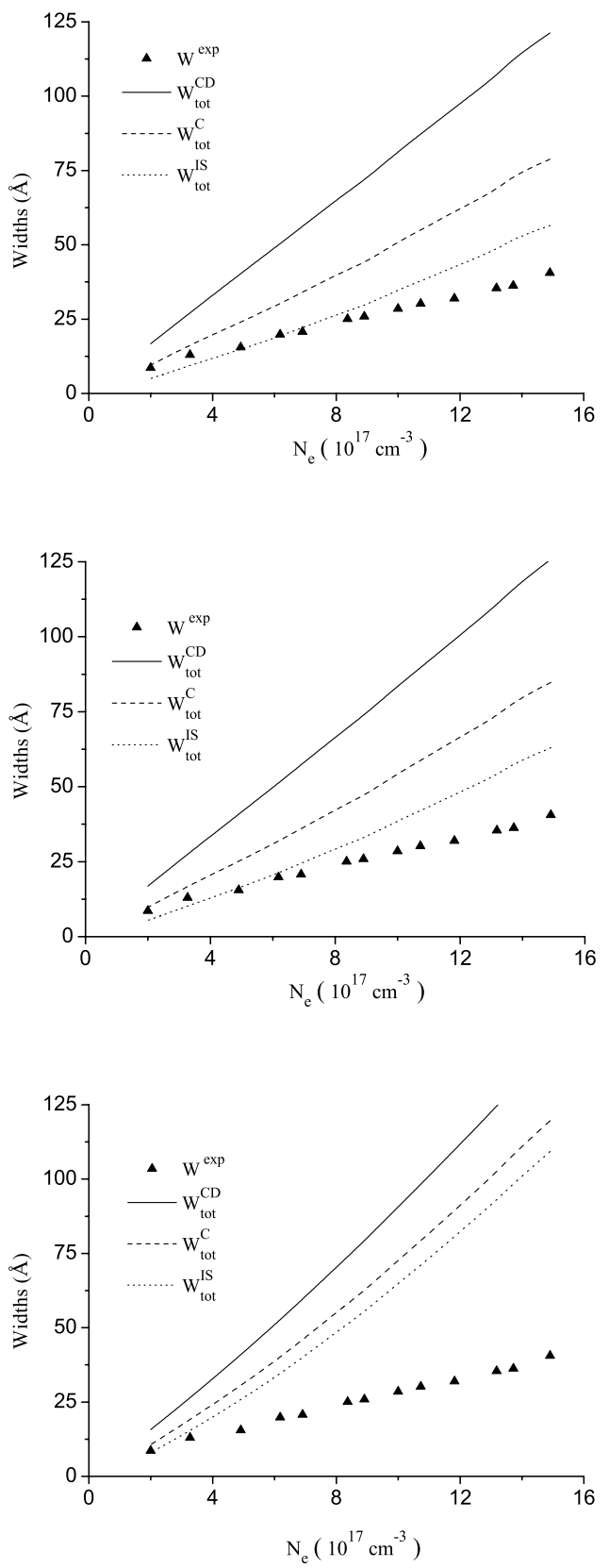

(c)

Fig. 3. Total widths $W_{\text {tot }}^{\mathrm{CD}}, W_{\mathrm{tot}}^{\mathrm{C}}$ and $W_{\text {tot }}^{\mathrm{IS}}$ obtained from the expression of Eq. (58) for the HeI $6678 \AA\left(2{ }^{1} \mathrm{P}^{\circ}-3^{1} \mathrm{D}\right)$ transition, where the associated micro-field distribution $W_{r}(\beta)$ is determined with: a) the Baranger \& Mozer's method (Baranger \& Mozer 1959; Mozer \& Baranger 1960), b) the Hooper's method (Hooper 1966, 1968a,b), and c) the AFF method (Potekhin et al. 2002). $W^{\text {exp }}$ is the experimental width relative to the same transition (Gauthier et al. 1981).

The different results may be improved by considering a hyperbolic trajectory. Such a trajectory would be more reliable for describing screening effects on the perturber motion colliding with a neutral atom, especially for very low collision energies in the neighborhood of the threshold (Jung 1994). However, for large impact parameters, the straight line trajectory can be used even when the emitter is an ion (Jung 2000). As we are interested only in neutral atom emitters for large impact parameters at relatively low energies (temperatures of the order of a few thousand
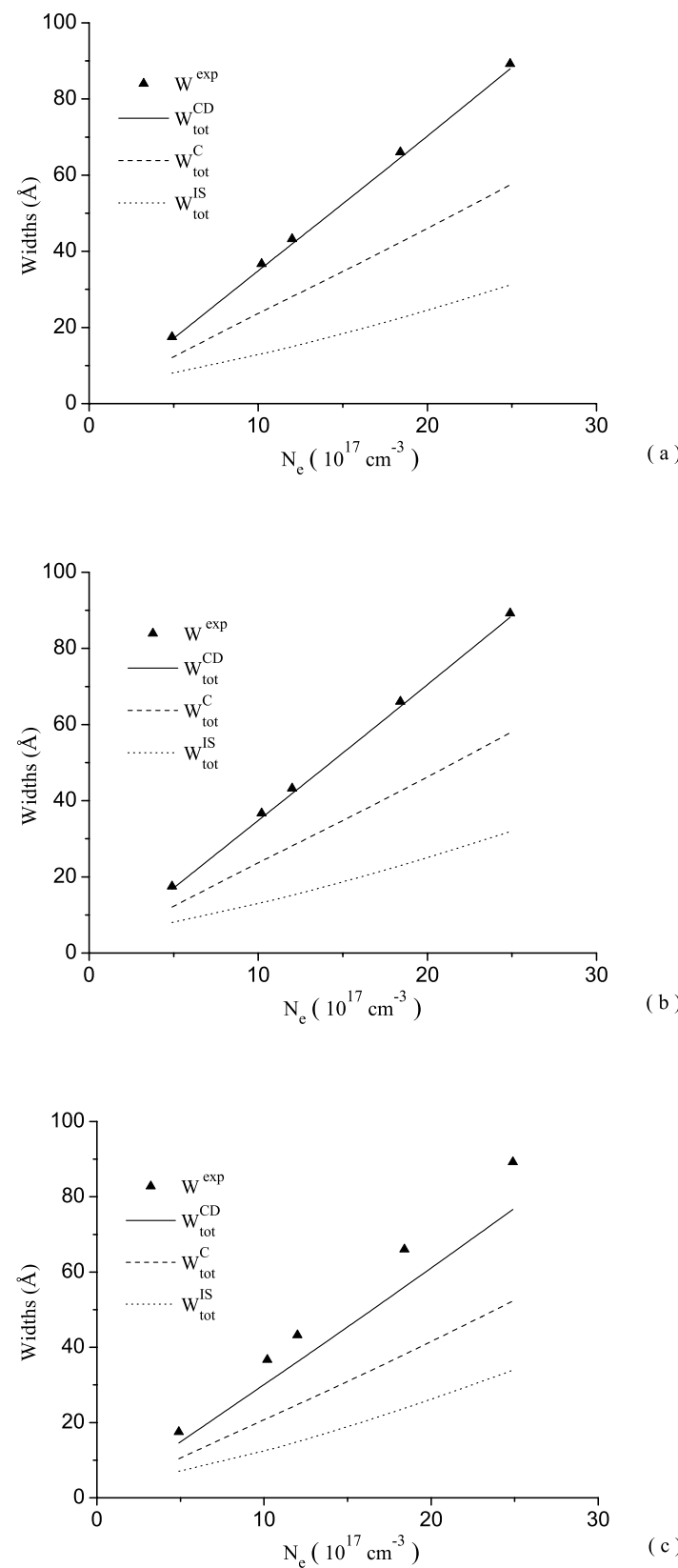

(c)

Fig. 4. Same as Fig. 3 for the HeI $5876 \AA\left(2^{3} \mathrm{P}^{\circ}-3^{3} \mathrm{D}\right)$ line studied in the same conditions of densities $N_{\mathrm{e}}$ and temperatures $T$ of the experiment of Bücher et al. (1995).

or few ten thousands degrees), we have neglected the plasma screening effects on the semi-classical straight line trajectory. The dynamic plasma screening effects on the atomic excitation process are found to be significant only for relatively high energy projectiles (Jung \& Yoon 2000b). The excitation cross section including screening effects is shown to decrease as the non-ideality factor $\gamma$ increases (Song \& Jung 2003; Jung 2000).

This study has qualitatively shown the importance of the electronic correlations for a non-ideal plasma. However, many refinements should be more deeply studied and introduced gradually with care, before trying to generalize our theoretical approach to other plasmas studies. 
Table 5. Total width ratios calculated for the considered HeI transitions, where the micro-field distribution $W_{r}(\beta)$ is determined with: (a) the Baranger \& Mozer's method (Baranger \& Mozer 1959; Mozer \& Baranger 1960); (b) the Hooper's method (Hooper 1966, 1968a,b); and (c) the AFF method (Potekhin et al. 2002). $W_{\text {tot }}^{\mathrm{G}}$ : total width obtained from the expression of Eq. (59) $-W_{\text {tot }}^{\mathrm{G}, *}$ : total width obtained by considering $r=0-W_{\mathrm{tot}}^{\mathrm{CD}}, W_{\mathrm{tot}}^{\mathrm{C}}$ and $W_{\mathrm{tot}}^{\mathrm{IS}}$ : total widths obtained from the expression of Eq. $(58)-W_{\text {tot,app }}^{\mathrm{CD}}, W_{\text {tot,app }}^{\mathrm{C}}$ and $W_{\text {tot,app }}^{\mathrm{IS}}$ : total widths approximated by the expressions of Eqs. (64)-(66).

\begin{tabular}{|c|c|c|c|c|c|c|c|c|c|c|}
\hline $\begin{array}{c}\frac{W_{\mathrm{tot}}^{\mathrm{G}}}{W_{\mathrm{tot}}^{\mathrm{CD}}} \\
(\%)\end{array}$ & $\begin{array}{c}\frac{W_{\mathrm{tot}}^{\mathrm{G}, *}}{W_{\mathrm{tot}}^{\mathrm{CD}}} \\
(\%) \\
\end{array}$ & $\begin{array}{c}\frac{W_{\text {tot,app }}^{\mathrm{CD}}}{W_{\text {tot }}^{\mathrm{CD}}} \\
(\%)^{(a)}\end{array}$ & $\begin{array}{c}\frac{W_{\text {tot,app }}^{\mathrm{CD}}}{W_{\text {tot }}^{\mathrm{CD}}} \\
(\%)^{(b)}\end{array}$ & $\begin{array}{c}\frac{W_{\text {tot,app }}^{\mathrm{CD}}}{W_{\text {tot }}^{\mathrm{CD}}} \\
(\%)^{(c)}\end{array}$ & $\begin{array}{l}\frac{W_{\text {tot,app }}^{\mathrm{C}}}{W_{\text {tot }}^{\mathrm{C}}} \\
(\%)^{(a)}\end{array}$ & $\begin{array}{l}\frac{W_{\text {tot,app }}^{\mathrm{C}}}{W_{\text {tot }}^{\mathrm{C}}} \\
(\%)^{(b)}\end{array}$ & $\begin{array}{c}\frac{W_{\text {tot,app }}^{\mathrm{C}}}{W_{\text {tot }}^{\mathrm{C}}} \\
(\%)^{(c)}\end{array}$ & $\begin{array}{c}\frac{W_{\text {tot,app }}^{\mathrm{IS}}}{W_{\text {tot }}^{\mathrm{IS}}} \\
(\%)^{(a)}\end{array}$ & $\begin{array}{c}\frac{W_{\text {tot,app }}^{\text {IS }}}{W_{\text {tot }}^{\text {IS }}} \\
(\%)^{(b)}\end{array}$ & $\begin{array}{c}\frac{W_{\text {tot,app }}^{\mathrm{IS}}}{W_{\mathrm{tot}}^{\mathrm{IS}}} \\
(\%)^{(c)}\end{array}$ \\
\hline \multicolumn{11}{|c|}{$\begin{array}{c}\left(2^{1} \mathrm{P}^{\circ}-3^{1} \mathrm{D}\right) \\
6678 \AA\end{array}$} \\
\hline 98.80 & 123.16 & 100.43 & 101.41 & 112.33 & 101.45 & 103.21 & 116.35 & 104.85 & 105.78 & 113.39 \\
\hline 98.89 & 124.65 & 100.17 & 99.87 & 98.93 & 99.92 & 99.74 & 98.75 & 99.59 & 99.58 & 99.03 \\
\hline 97.92 & 127.62 & 99.83 & 100.44 & 102.87 & 100.58 & 100.82 & 103.04 & 101.18 & 101.30 & 102.49 \\
\hline 97.80 & 129.81 & 100.03 & 100.57 & 103.62 & 100.71 & 101.11 & 103.94 & 101.59 & 101.79 & 103.32 \\
\hline 97.72 & 130.47 & 99.99 & 100.34 & 102.16 & 100.51 & 100.73 & 102.24 & 101.09 & 101.15 & 102.12 \\
\hline 97.58 & 131.86 & 100.00 & 100.20 & 100.58 & 100.21 & 100.33 & 100.52 & 100.37 & 100.40 & 100.61 \\
\hline 97.66 & 133.38 & 100.39 & 100.52 & 103.80 & 100.76 & 101.08 & 104.17 & 101.39 & 101.69 & 103.21 \\
\hline 97.42 & 132.73 & 99.86 & 99.64 & 97.36 & 99.67 & 99.30 & 97.07 & 99.12 & 98.96 & 97.86 \\
\hline 97.30 & 133.25 & 99.82 & 99.61 & 97.13 & 99.60 & 99.15 & 96.88 & 98.96 & 98.76 & 97.60 \\
\hline 97.34 & 134.01 & 99.90 & 99.44 & 95.83 & 99.32 & 98.78 & 95.63 & 98.45 & 98.19 & 96.55 \\
\hline 97.26 & 135.27 & 100.04 & 99.44 & 96.55 & 99.37 & 98.97 & 96.46 & 98.63 & 98.48 & 97.22 \\
\hline 96.95 & 133.89 & 99.38 & 98.59 & 91.18 & 98.36 & 97.35 & 90.77 & 96.75 & 96.23 & 92.96 \\
\hline 97.09 & 135.83 & 99.89 & 99.06 & 94.44 & $\begin{array}{c}98.87 \\
\left(2^{3} \mathrm{P}^{\circ}-3^{3} \mathrm{D}\right)\end{array}$ & 98.31 & 94.33 & 97.79 & 97.58 & 95.58 \\
\hline & & & & & $5876 \AA$ & & & & & \\
\hline 101.36 & 109.14 & 100.07 & 100.01 & 100.06 & 99.92 & 99.95 & 100.12 & 100.17 & 100.20 & 101.45 \\
\hline 101.21 & 110.78 & 99.92 & 99.89 & 99.74 & 100.07 & 100.02 & 99.59 & 99.66 & 99.53 & 97.15 \\
\hline 101.13 & 111.32 & 99.92 & 100.24 & 100.70 & 100.30 & 100.29 & 101.05 & 100.13 & 100.31 & 100.69 \\
\hline 101.30 & 112.96 & 100.17 & 100.08 & 100.90 & 100.07 & 100.24 & 101.52 & 100.76 & 100.85 & 103.60 \\
\hline 101.20 & 113.47 & 99.92 & 99.75 & 98.46 & 99.61 & 99.44 & 97.53 & 99.19 & 99.00 & 96.80 \\
\hline
\end{tabular}

Table 6. Parameters $\left(a^{\mathrm{CD}}, b^{\mathrm{CD}}\right),\left(a^{\mathrm{C}}, b^{\mathrm{C}}\right)$ and $\left(a^{\mathrm{IS}}, b^{\mathrm{IS}}\right)$ of the expressions of Eqs. (64)-(66), calculated for the considered HeI transitions, where the micro-field distribution $W_{r}(\beta)$ is determined with: (a) the Baranger \& Mozer's method (1959; 1960), (b) the Hooper's method (Hooper 1966; $1968 \mathrm{a}, \mathrm{b})$, and (c) the AFF method (2002). For the sake of comparison, we give also in (d) the values of $a^{\mathrm{CD}}$ and $b^{\mathrm{CD}}$ defined in the expression of Eq. (59) of Griem $(1962,1974)$.

\begin{tabular}{cccccccc}
\hline \hline Transition & Models & $a^{\mathrm{CD}}$ & $b^{\mathrm{CD}}$ & $a^{\mathrm{C}}$ & $b^{\mathrm{C}}$ & $a^{\mathrm{IS}}$ & $b^{\mathrm{IS}}$ \\
\hline$\left(2^{1} \mathrm{P}^{\circ}-3^{1} \mathrm{D}\right)$ & (a) & -0.8275 & 1.5153 & -1.0433 & 1.6831 & -0.5560 & 1.5275 \\
$6678 \AA$ & (b) & -0.0885 & 1.1303 & 0.3223 & 0.9744 & 0.4179 & 1.1065 \\
& (c) & 7.7626 & -3.4044 & 8.5954 & -3.3916 & 6.8722 & -1.6409 \\
& (d) & -1.3125 & 1.75 & - & - & - & - \\
$\left(2^{3} \mathrm{P}^{\circ}-3^{3} \mathrm{D}\right)$ & (a) & -0.6395 & 1.4135 & -0.1114 & 1.2334 & 1.6013 & 0.6352 \\
$5876 \AA$ & (b) & 0.2977 & 1.0317 & 0.5720 & 0.9681 & 2.7525 & 0.2069 \\
& (c) & 5.8749 & -1.9181 & 8.8274 & -2.8408 & 16.6070 & -5.4567 \\
\hline
\end{tabular}

Acknowledgements. This work has been supported by the cooperation between the French CNRS and the Tunisian DGRSRT. We wish to thank the referee for very helpful comments. HBC is very grateful to Dr. N. Helali (Group of Complex Dynamic Systems (GCDS) 12.32) for his help during this work, especially for the development of the numerical codes which compute the line width in a nonideal plasma by considering the new semi-classical collision functions and the quasistatic ionic contribution.

\section{References}

Antia, H. M. 1993, ApJS. Ser., 84, 101

Baranger, M. 1958a, Phys. Rev. A, 111, 481

Baranger, M. 1958b, Phys. Rev. A, 111, 494

Baranger, M. 1958c, Phys. Rev. A, 112, 855

Baranger, M., \& Mozer, B. 1959, Phys. Rev., 115, 521

Baranger, M., \& Mozer, B. 1960, Phys. Rev., 118, 626
Baranger, M. 1962, in Atomic and Molecular Processes, ed. D. R. Bates (New York: Academic Press)

Ben Chaouacha, H., Ben Nessib, N., \& Sahal-Bréchot, S. 2004, A\&A, 419, 771 Ben Chaouacha, H., Ben Nessib, N., \& Sahal-Bréchot, S. 2005, A\&A, 433, 1153 Ben Nessib, N., Ben Lakhdar, Z., \& Sahal-Bréchot, S. 1996, Phys. Scr., 54, 608 Ben Nessib, N., Sahal-Bréchot, S., \& Ben Lakhdar, Z. 1997, A\&A, 324, 799 Bücher, S., Glenzer, S., Wrubel Th., \& Kunze, H.-J. 1995, J. Quant. Spectrosc. Radiat. Transf., 54, 73

Cunto, W., Mendoza, C., Ochsenbein, F., \& Zeippen, C. J. 1993, A\&A, L5, 275 Debye, P., \& Hückel, E. 1923, Z. Phys., 25, 185

Dimitrijević, M. S., \& Sahal-Bréchot, S. 1984a, A\&A, 136, 289

Dimitrijević, M. S., \& Sahal-Bréchot, S. 1984b, J. Quant. Spectrosc. Radiat. Transf., 31, 301

Dimitrijević, M. S., \& Sahal-Bréchot, S. 1985, Phys. Rev. A, 31, 316

Dimitrijević, M. S., \& Sahal Bréchot, S. 1990, A\&A, 82, 519

Feautrier, N. 1968, Annales d'Astrophysique, 31, 305

Gauthier, J.-C., Geindre, J.-P., Goldbach, C., et al. 1981, J. Phys. B, At. Mol. Phys., 14, 2099 
Griem, H. R. 1962, Phys. Rev., 128, 515

Griem, H. R., Baranger, M., Kolb, A. C., \& Ortel, G. 1962, Phys. Rev., 125, 177 Griem, H. R. 1964, Plasma Spectroscopy (New York: Mc-Graw Hill Book Go.), USA

Griem, H. R. 1974, Spectral Line Broadening by Plasmas (New York (USA) and London (UK): Academic Press Inc.)

Günther, K., Hess, H., \& Radtke, R. 1985, Iv. Papers, 17th ICPIG, 120

Gutierrez, F. A., Jouin, H., \& Cormier, E., J. Quant. Spectrosc. Radiat. Transf., 51,665

Holtsmark, J. 1919, Ann. Physik, 58, 577

Hooper, C. F., Jr. 1966, Phys. Rev., 149, 77

Hooper, C. F., Jr. 1968a, Phys. Rev., 165, 215

Hooper, C. F., Jr. 1968b, Phys. Rev., 169, 193

Iglesias, C. A., Lebowitz, J. L., \& MacGowan, D. 1983, Phys. Rev. A, 28, 1667

Iglesias, C. A., \& Lebowitz, J. L. 1984, Phys. Rev. A, 30, 2001

Iglesias, C. A. 2000, J. Quant. Spectrosc. Radiat. Transf., 65, 303

Jung, Y.-D. 1994, Phys. Rev. A, 50, 3895

Jung, Y.-D. 2000, Eur. Phys. J. D, 11, 291

Jung, Y.-D., \& Yoon, J.-S. 2000a, ApJ, 530, 1090

Jung, Y.-D., \& Yoon, J.-S. 2000b, Phys. Scr., 62, 46

Konjević, N., Lesage, A., Fuhra, J. R., \& Wiese, W. L. 2002, J. Phys. Chem. Ref. Data, 31,819

Margenau, H., \& Lewis, M. 1959, Rev. Mod. Phys., 31, 569

Mayer, J. E., \& Mayer, M. G. 1940, Statistical Mechanics (New York: John Wiley, \& Sons, Inc.), Chap. 13
Mozer, B., \& Baranger, M. 1960, Phys. Rev., 118, 626

Omar, B., Günter, S., Wierling, A., \& Röpke, G. 2006, Phys. Rev. E, 73,1

Poquerusse, A. 2000, Eur. Phys. J. D, 10, 307

Potekhin, A. Y., Chabrier, G., \& Gilles, D. 2002, Phys. Rev. E, 65, 1

Sahal-Bréchot, S. 1969a, A\&A, 1, 91

Sahal-Bréchot, S. 1969b, A\&A, 2, 322

Salzmann, D., \& Szichman, H. 1987, Phys. Rev. A, 35, 807

Scheibner, K., Weisheit, J. C., \& Lane, N. F. 1987, Phys. Rev. A, 35, 1252

Seaton, M. J. 1962, Proc. Phys. Soc., 79, 1105

Sidney, A., Freundenstein, \& Cooper, J. 1987, ApJ, 224, 1079

Song, M.-Y., \& Jung, Y.-D. 2003, J. Phy. B, At. Mol. Opt. Phys., 36, 2119

Stewart, J. C., \& Pyatt, K. D. 1966, ApJ, 144, 1203

The Opacity Project Team 1995, the Opacity Project, Vol. 1 (Bristol, UK: Institute of Physics Publ.)

Vidal, C. R., Cooper, J., \& Smith, E. W. 1971, Unified Theory Calculations of Stark Broadened Hydrogen Lines Including Lower State Interactions, National Bureau of Standards Monograph, 120

Vitel, Y., Mokhtari, A., \& Skowronek, M. 1990, J. Phy. B, At. Mol. Opt. Phys., 23,651

Weisheit, J. C. 1984, in Applied Atomic Collision Physics, ed. H. S. W. Massey (New York: Academic), Vol. II, 441

Zeippen, C. J. 1995, Phys. Scr. T, 58, 43 